\title{
Alexander Konyukhov \\ Wave cancellation conditions for the double impact of finite duration in an arbitrary structure
}

Received: 11 July 2019 / Revised: 12 December 2019 / Published online: 21 May 2020

(C) The Author(s) 2020

\begin{abstract}
Resonance phenomena in impacting systems can be defined as an amplitude increasing during periodically applied impacts. The wave cancellation phenomenon is defined as application of certain conditions to cancel the wave fully. The double impact system is defined as the application of the first impact with a certain duration $\tau$ and then the application of a counter impact in a certain time $\tau_{1}$ such that the vibrations caused by the first impact are fully disappearing. In the current contribution this phenomenon is first studied for the simplest 1D bar vibration. The response function is introduced as a characteristic for such a phenomenon and, by studying its properties, it is possible to find both an impact duration time $\tau$ and an application time $\tau_{1}$ for the counter impact leading to the wave cancellation. The result is generalized for any arbitrary homogeneous linear non-dissipative mechanical structure described by a semi-elliptic operator $L u$. The counter impact can be determined in the same way as in the opposite direction. This general result is numerically illustrated for various operators $L u$ possessing relatively simple analytical solutions: for a simply supported and a clamped Bernoulli beam, for a fixed membrane and for a Kirchhoff plate. Three potential applications are discussed at the end: a set of verification examples for further analysis of time integration numerical schemes with the energy conservation property; straightforward transfer of cancellation conditions for the double impact to any convenient numerical method in mechanics, e.g. finite element method, iso-geometric method etc.; application of the result in engineering design of impacting devices (hammering etc.) in order to prevent recoil.
\end{abstract}

\section{Introduction}

The structural resonance phenomenon is well known as an increase of the vibration amplitude if the frequency of the exciting force is approaching the eigenfrequency of the structure. In non-dissipative system it leads to a linear increase of the amplitude. Many monographs in dynamics describes this example, see e.g. more earlier [18] and more recent [29]. Various aspects of the impact in mechanical systems are studied in the monographs $[2,4,10,12,15,17]$. Various aspects of the resonance phenomena are investigated in $[8,23,24,27$, $31]$ and including impact in [16].

An opposite to the structural resonance is the wave cancellation phenomenon-application of such conditions including active force, moment etc. when the first wave produced in the structure is canceled fully or is strongly decreased. Conditions allowing to strongly decrease the first wave came into practice within the last decades as the so-called active noise control in acoustic. Chung and Crocker [6] proposed the acoustic wave cancellation induced by the monopole radiator. The acoustic wave was induced by the vibration of the simply supported beam. Approximated conditions have been found for the dipole radiators. Yang [36] consider vibration of simple beams due to trains, in which moving loads have been represented via compositions of Dirac delta functions and Heaviside functions. Both wave cancellation and resonance conditions have been 
derived in closed analytical form. Also, comparisons of the results with finite element solutions have been performed. The wave cancellation conditions have been studied in closed form for discrete two mass points system by Dingyue et al. [9]. Yang et al. [35] considered the mechanism of the resonance and cancellation for train-induced vibrations in a bridge. The bridge is modeled by the simply supported beam on the elastic foundation. Loads are represented by the series of Delta functions pulse moving loads. The exact condition for the moving load is found to cause ether resonance or cancellation conditions. Yau et al. [37] implemented tuned mass dampers (TMD) for cable-stayed bridges in order to decrease the vibration amplitude during the passing of a high speed train as an example of combination of active and passive control. It is shown that the larger the number of stay cables of a cable-stayed bridge, the smaller the impact response of the bridge. The moving load is represented by the pulse Dirac function. Among the active vibration control in structural systems one can select rather special cases allowing a closed form for the wave cancellation. Zhang and Chen [38] have found the wave cancellation condition for a moving string being implemented as an additional tensioner. The effect is shown due to the the additional tensioner for the string rather than due to superposition of waves. Zhang et al. [39] considered a piezoelectric actuator for the wave cancellation induced in a simply supported cylindrical Kirchhoff type shell. The solution was formulated in analytical form via sinusoidal harmonics. Schoeftner and Juergen in [26] illustrated for the Bernoulli-Euler beam, that bonding piezoelectric layers with attached electric circuits can be used as a passive method for wave cancellation as a single point control (SPC). Both semi-analytical numerical computations and finite element computations in ANSYS were employed for the model verification. The active control of noise and vibration methods were widely developed and implemented in various fields of engineering practice, see the review in Kuo and Morgan [20] and more recent monograph of Colin et al [13]. Auersch in [1] proposed a combined finite-element boundary-element method (FEBEM) where the response of the infinitely long plate is calculated by a numerical integration in the frequency-wavenumber domain. Conditions for the wave reductions were shown. Vankata Rao et al. [32] applied the finite element method to model Mindlin type plates and studied piezoelectric actuators for the vibration control. Futhazar et al. [11] proposed active cloaking which allows to cancel waves in a specific finite domain of the infinite Kirchhoff plate. The sources as well as scatters are pulsed forces represented by Delta functions. Teo and Fleming in [30] developed further an active damping control, the integral force feedback method (IFF). It is shown experimentally that the improved IFF method can achieve arbitrary damping for any mechanical system by introducing a feed-through term. There are also recent active noise control methods. The reconstruction of forces generated by multiple impacts occurring in linear elastic structures has been considered in [25] in which, in addition, statistical methods have been involved. The wave boundary control method of large net structures was considered by Zuo et al. [40]. In this article a net structure was modeled as a set of intersecting orthogonal strings, described by $1 \mathrm{D}$ wave equations. This representation of the net structure allows to derive various control laws in the closed forms. Lee et al. [21] described experimental investigations demonstrating that the incident beams of ultrasonics waves can be canceled by using an elastic phononic crystal prism. Huang and $\mathrm{Xu}$ in [14] proposed an active control method used for electromagnetic wave cancellation based on generation of a periodic signal in order to cancel the radar target echo. Three conditions should be satisfied for the cancellation. The source signal is represented by the Delta function. Lu et al. [22] described resonance and cancellation conditions caused by the equidistant moving pulse loads in a pile-supported viaduct. Spans of the bridge are modelled as a simply supported beam loaded by the moving pulse load. It has been found that if the time lag between two neighboring moving loads is satisfying certain conditions with regards to the resonance frequency then either resonance or cancellation conditions may occur.

The novelty of the current contribution is the formulation of the wave cancellation conditions for the double impact system for an arbitrary structure. The double impact system is defined as a mechanical structure in which the first impact of finite duration enforces the vibrations in a structure and the second impact leads to cancellation of the vibrations in the whole structure. The second impact is specially constructed with the help of the introduced response function such that the vibrations induced by the first impact will be fully canceled.

Properties of the response function allows to construct the second impact satisfying the wave cancellation condition for any arbitrary mechanical structure subjected to the double impact problem. The main characteristics of the double impact problem are:

- The first impact of final duration $\tau$ is forcing the structure to vibrate. The energy is supplied and remains constant.

- The second impact of the same duration $\tau$ is applied at the specially determined time $\tau_{1}$ depending on the structure, namely of eigenfrequencies. This time can be selected a-priory larger than any given real number $t_{\text {Large }}$, i.e. $\tau_{1}>t_{\text {Large }}$. The second impact can be applied in the same direction as well as in the opposite 


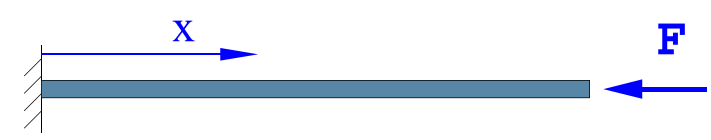

Fig. 1 Bar loaded with longitudinal force $F$ of duration $\tau_{1}$

direction and necessarily leads to the wave cancellation caused by the first impact. The whole structure is standing still and the energy is becoming zero.

The article is organized as follows:

1. The double impact problem is shown, first, for the simple 1D bar problem in the closed form solution, in which the response function is introduced.

2. All properties of the response function are studied. This allows to formulate wave cancellation conditions for the second impact.

3. The wave cancellation conditions for the double impact problem are studied via the response function for an arbitrary linear dynamics problem described with a linear semi-elliptic operator $L u$ :

$$
\rho \frac{\partial^{2} u}{\partial t^{2}}=L u(x)+q(x, t), \quad x \in \Omega
$$

4. Since the general result is applicable for a large number of mechanical problems-e.g., various beam and shell models for engineering structures, wave propagation in 2D and 3D continuum etc.- the double impact is shown selectively for models possessing relatively simple analytical solutions:

- a simply supported Bernoulli beam;

- a cantilever Bernoulli beam;

- a membrane;

- a Kirchhoff beam.

\section{Double impact in 1D bar}

Here, we consider the statement of the double impact problem for a 1D bar. The closed form solution is obtained in the form of generalized Fourier series via the combination of several solution methods for partial differential equations (PDE), see [13,33,34], and includes the following steps:

- Fourier series method in order to obtain solution of the homogeneous PDE;

- Fourier series method to in order obtain the fundamental solution of PDE, satisfying all boundary conditions;

- Representation of the impact of duration $\tau$ via the Heaviside functions for the non-homogeneous ODE;

- Solution of the non-homogeneous PDE in the form of convolution with the fundamental solution of the PDE.

The first two methods are standard methods for graduate courses and are presented in Appendix A for completeness. The combination of the last two methods leads to the response function which is studied in detail in order to construct wave cancellation conditions caused by the second impact.

\subsection{Statement of the double impact problem for the 1D-bar}

Consider a homogeneous bar with length $L$, with a constant cross section area $A$, density $\rho$ and Young's module of elasticity $E$. The bar is fixed on the left side, and the right end is free, see Fig. 1. At the beginning, the bar is in an idle state.

Motion of the bar-longitudinal vibrations $-u(x, t)$ satisfies the following partial differential equation (PDE):

$$
\rho A \frac{\partial^{2} u}{\partial t^{2}}-E A \frac{\partial^{2} u}{\partial x^{2}}=q(x, t)
$$

with corresponding boundary conditions

$$
u(0, t)=0,- \text { fixed end, } \quad \frac{\partial u}{\partial x}(L, t)=0, \quad \forall t>0-\text { free end },
$$


and initial conditions

$$
\begin{cases}u(x, 0)=0, & \text { for }[0 \leq x \leq L]-\text { no initial displacements } \\ \frac{\partial u}{\partial t}(x, 0)=0, & \text { for }[0 \leq x \leq L]-\text { no initial velocity }\end{cases}
$$

The first impact of the finite duration is defined as follows: at time $t=0$ the bar is loaded at the free end with the constant force $F$ and time duration of $\tau$. The first impact is causing the longitudinal vibration $u(x, t)$ and wave distribution starting from the free end. In order to describe this impact, the distributed along the line external force $q(x, t)$ with physical dimensions $\left[\mathrm{N} \mathrm{m}^{-1}\right]$ is formulated via the Dirac and Heaviside functions. The second impact with the force $F_{1}$ and time duration of $\tau$ will be applied at time $\tau_{1}$. The double impact is formulated as a combination of the first and the second impact of the same duration $\tau$.

\subsubsection{Formulation of the various impacts of duration $\tau$ via the Dirac and Heaviside functions for the non-homogeneous $O D E$}

The distributed force $q(x, t)$ can be specially configured in order to describe the impact force $F$ applied at the end of the bar $x=L$ using the Dirac function $\delta(x)$ for the longitudinal coordinate $x$. The time duration $\tau$ for the application of the impact force $F$ is implied via the composition of Heaviside functions $H(t)-H(t-\tau)$. The expression for such an external force is written as

$$
q_{\tau}(x, t)=F \delta(x-L)[H(t)-H(t-\tau)] .
$$

The impact force of zero time duration can be written via the Dirac function for the time variable $\delta(t)$ as follows:

$$
q_{\delta}(x, t)=F \delta(x-L) \frac{\mathrm{d} H(t)}{\mathrm{d} t}=F \delta(x-L) \delta(t) .
$$

The double impact is constructed as a composition of the first impact $F$ in Eq. (4) at time $t=0$ and the second impact $F_{1}$ of duration $\tau$ at time $\tau_{1}$ :

$$
q_{\left(F F_{1}\right)}(x, t)=F \delta(x-L)[H(t)-H(t-\tau)]+F_{1} \delta(x-L)\left[H\left(t-\tau_{1}\right)-H\left(t-\tau-\tau_{1}\right)\right] .
$$

\subsubsection{Solution of the non-homogeneous PDE in the form of convolution with the fundamental solution of PDE}

The solution of the non-homogeneous PDE will be obtained if we solve the corresponding non-homogeneous ODE in Eq. (97) with an arbitrary right side. The solution-following steps 1, 2, 4 in Sect. 2-is written in the form of Fourier series employing the standard results represented in Appendix A. Another similar technique is employing the Duhamel integral and is widely used in control mechanics, see: $[13,22,35]$ :

$$
u(x, t)=\sum_{n=0}^{\infty} T_{n}(t) X_{n}(x)=\sum_{n=0}^{\infty} T_{n}(t) \sin \left(\frac{(2 n+1) \pi}{2 L} x\right),
$$

where

$$
T_{n}(t)=\frac{\sin \left(\omega_{n} t\right)}{\omega_{n}} H(t) * \frac{\left\langle q(x, t) \cdot X_{n}(x)\right\rangle}{\rho A\left\|X_{k}(x)\right\|^{2}} H(t) ;
$$

here the eigenfrequencies $\omega_{n}$ are written as

$$
\omega_{n}=c \lambda_{n}=\sqrt{\frac{E}{\rho}} \frac{[(2 n+1) \pi]}{2 L}, \quad n=0,1,2, \ldots
$$

In order to study the double impact problem and formulate wave cancellation conditions, we introduce a response function. 
2.2 Response function and its properties

First, we introduce $n$th eigen period value for the structure $P_{n}$, based on its eigenfrequency $\omega_{n}$.

Definition 1 the $n$th eigenperiod is defined as

$$
P_{n}=\frac{2 \pi}{\omega_{n}} .
$$

Then, we introduce the response function (see also in [13]) in order to construct the second counter impact satisfying the wave cancellation condition.

Definition 2 The response function is defined as the convolution of the fundamental solution in Eq. (96) with a single unit impact in Eq. (4):

$$
\begin{aligned}
\Phi_{n}\left(t, \tau, \tau_{1}\right)= & T_{f}(t) *\left[H\left(t-\tau_{1}\right)-H\left(t-\tau-\tau_{1}\right)\right] \\
= & \frac{\sin \left(\omega_{n} t\right)}{\omega_{n}} H(t) *\left[H\left(t-\tau_{1}\right)-H\left(t-\tau_{1}-\tau\right)\right] \\
= & \frac{1}{\omega_{n}} \int_{-\infty}^{\infty}\left[\sin \left(\omega_{n} u\right) H(u)\left[H\left(t-\tau_{1}-u\right)-H\left(t-u-\tau_{1}-\tau\right)\right]\right] \mathrm{d} u \\
= & \frac{1}{\omega_{n}^{2}}\left(\left[1-\cos \left(\omega_{n}\left(t-\tau_{1}\right)\right)\right] H\left(t-\tau_{1}\right)\right. \\
& \left.-\left[1-\cos \left(\omega_{n}\left(t-\tau_{1}-\tau\right)\right)\right] H\left(t-\tau_{1}-\tau\right)\right) .
\end{aligned}
$$

The response function is, therefore, the solution of the single impact problem in terms of functions $\Phi_{n}\left(t, \tau, \tau_{1}\right)=T_{n}(t)$ (see Eq. (7)) for the unit force $F=1$ with time duration $\tau$ and applied at time $t=\tau_{1}$.

Superposition of two response functions is reflecting the double impact situation. Some properties are necessary to study this situation for various structures. All properties are defined after the application of the second impact, i.e. for $t>\tau_{1}+\tau$.

Property 1 (Sum of two impacts in the same direction) For $t>\tau_{1}+\tau$ the following transformation is valid:

$$
\begin{aligned}
\Phi_{n}(t, \tau, 0)+\Phi_{n}\left(t, \tau, \tau_{1}\right)= & \frac{1}{\omega_{n}^{2}}\left[\left[1-\cos \left(\omega_{n} t\right)\right]-\left[1-\cos \left(\omega_{n}(t-\tau)\right)\right]\right. \\
& \left.+\left[1-\cos \left(\omega_{n}\left(t-\tau_{1}\right)\right)\right]-\left[1-\cos \left(\omega_{n}\left(t-\tau_{1}-\tau\right)\right)\right]\right] \\
= & \frac{1}{\omega_{n}^{2}}\left[\cos \left(\omega_{n}(t-\tau)\right)-\cos \left(\omega_{n} t\right)\right. \\
& \left.+\cos \left(\omega_{n}\left(t-\tau_{1}-\tau\right)\right)-\cos \left(\omega_{n}\left(t-\tau_{1}\right)\right)\right] \\
& \text { the sum of cosines formula is applied } \\
& \text { to the } 1 \text { st plus 3rd and 2nd plus 4th terms } \\
= & \frac{2}{\omega_{n}^{2}}\left[\cos \left(\omega_{n}\left(t-\tau-\frac{\tau_{1}}{2}\right)\right)-\cos \left(\omega_{n}\left(t-\frac{\tau_{1}}{2}\right)\right)\right] \cos \frac{\omega_{n} \tau_{1}}{2} \\
& \text { the difference of cosines formula leads to } \\
= & \frac{4}{\omega_{n}^{2}}\left[\sin \left(\omega_{n} \frac{2 t-\tau-\tau_{1}}{2}\right) \sin \left(\frac{\omega_{n} \tau}{2}\right)\right] \cos \frac{\omega_{n} \tau_{1}}{2} .
\end{aligned}
$$

Property 2 (Sum of two impacts in the opposite direction)

For $t>\tau_{1}+\tau$ the following transformation is valid:

$$
\begin{aligned}
\Phi_{n}(t, \tau, 0)-\Phi_{n}\left(t, \tau, \tau_{1}\right)= & \frac{1}{\omega_{n}^{2}}\left[\left[1-\cos \left(\omega_{n} t\right)\right]-\left[1-\cos \left(\omega_{n}(t-\tau)\right)\right]\right. \\
& \left.-\left[1-\cos \left(\omega_{n}\left(t-\tau_{1}\right)\right)\right]-\left[1-\cos \left(\omega_{n}\left(t-\tau_{1}-\tau\right)\right)\right]\right]
\end{aligned}
$$


applying the corresponding

trigonometric transformations

$$
=\frac{4}{\omega_{n}^{2}}\left[\cos \left(\omega_{n} \frac{4 t-\tau-2 \tau_{1}}{4}\right) \sin \left(\frac{\omega_{n} \tau}{2}\right)\right] \sin \frac{\omega_{n} \tau_{1}}{2} .
$$

Property 3 (Zeros of the response function. Self cancellation condition for a single impact)

If the duration of the impact is equal to an integer number of eigenperiods,

$$
\tau=k P_{n}, \quad k, n=1,2, \ldots
$$

then the response function in Eq. (10) after time $t>\tau_{1}+\tau$ is becoming identically zero.

This statement is resulting from the term $\sin \frac{\omega_{n} \tau}{2}$ which is presented in both Eqs. (11) and (12).

$$
\sin \frac{\omega_{n} \tau}{2}=\sin \frac{k \omega_{n} P_{n}}{2}=\sin \frac{k \omega_{n} 2 \pi}{2 \omega_{n}}=\sin (\pi k)=0 .
$$

Thus, if the time duration $\tau$ for the single impact is proportional to an integer number of eigenperiods, then after the time $t>\tau+\tau_{1}$ vibrations will be canceled. This is valid, of course, for the double impact—simply after each impact vibrations will be canceled. This self cancellation is observed for the time duration $\tau$ at least larger or equal to the first eigenperiod: $\tau \geq T_{1}$ with regard to Eq. (13).

Remark 1 If the duration of the impact is shorter then the first eigenperiod $P_{1}$ and the second impact is applied at the time $\tau_{1}$ proportional to the $k$ th eigenperiod,

$$
\tau_{1}=\alpha P_{k}, \quad k=1,2, \ldots, \alpha \in \Re,
$$

then it is possible to find such a time $\tau_{1}$, when the second impact of the same duration $\tau$ should be applied, such that the sum of the response functions is becoming identically zero. The exact definition of $\alpha$ now depends on the situation: either two impacts in the same direction Eq. (11), or two impacts in the opposite direction Eq. (12) are considered. According to Eq. (15), this time $\tau_{1}$ can be larger than an arbitrary given real number.

Property 4 (Zeros of the response function. Duration of the impact is less then the first eigenperiod $P_{1}$. Sum of two impacts in the same direction)

In this case it is required from Eq. (11) that the following term should be zero:

$$
\cos \frac{\omega_{n} \tau_{1}}{2}=0
$$

which leads to

$$
\pi \alpha \frac{\omega_{n}}{\omega_{k}}=\pi \frac{2 m+1}{2} \Longrightarrow \alpha \frac{\omega_{n}}{\omega_{k}}=\frac{2 m+1}{2} .
$$

If the parameter $\alpha$ is chosen such that Eq. (17) can be resolved in natural numbers, then the second impact in the same direction applied at the time $\tau_{1}$ from Eq. (15) is causing the zeros of the response function.

Property 5 (Zeros of the response function. Duration of the impact is less then the first eigen period $P_{1}$. Sum of two impacts in the opposite direction)

In this case it is required from Eq. (12) that the following term should be zero:

$$
\sin \frac{\omega_{n} \tau_{1}}{2}=0
$$

which leads to

$$
\pi \alpha \frac{\omega_{n}}{\omega_{k}}=\pi m \Longrightarrow \alpha \frac{\omega_{n}}{\omega_{k}}=m
$$

If the parameter $\alpha$ is chosen such that Eq. (19) can be resolved in natural numbers, then the second impact in the opposite direction applied at the time $\tau_{1}$ from Eq. (15) is causing the zeros of the response function.

Corollary 1 The wave cancellation conditions for the double impact problem for $1 D$ bar can be formulated as follows: 
1. If the second impact of the same duration $\tau$ applied in the same direction at the time $\tau_{1}$, satisfying Eqs. (15) and (17), then the wave induced by the first impact is fully cancelled.

2. If the second impact of the same duration $\tau$ applied in the opposite direction at the time $\tau_{1}$, satisfying Eqs. (15) and (19), then the wave induced by the first impact is fully cancelled.

3. It is obvious, that the both cancellation conditions remain valid also for impact of zero time duration, i.e. described via the Dirac function $\delta(t)$.

Solution of Eqs. (17) and (19) depends on the structure of the eigenvalues in a certain case and will be studied by examples.

\subsection{Solution for various impact problems}

Let us study in details all impact situations for 1D bar. Consider analytical solutions using the definition of the response function in terms of time dependent functions $T_{n}(t)$ for the following cases:

1. A single impact of duration $\tau$, see right side in Eq. (4):

$$
\begin{aligned}
T_{n}(t) & =\frac{\sin \left(\omega_{n} t\right)}{\omega_{n}} H(t) * \frac{\left\langle q(x, t) \cdot X_{n}(x)\right\rangle}{\rho A\left\|X_{k}(x)\right\|^{2}} \\
& =\frac{\sin \left(\omega_{n} t\right)}{\omega_{n}} H(t) * F\left(\delta(x-L) \cdot X_{n}(x)\right) \frac{[H(t)-H(t-\tau)]}{\rho A\left\|X_{k}(x)\right\|^{2}} \\
& =F \frac{X_{n}(L)}{\rho A\left\|X_{k}(x)\right\|^{2}} \frac{\sin \left(\omega_{n} t\right)}{\omega_{n}} H(t) *[H(t)-H(t-\tau)] \\
& =F \frac{X_{n}(L)}{\rho A\left\|X_{k}(x)\right\|^{2}} \Phi_{n}(t, \tau, 0)
\end{aligned}
$$

The eigenfrequencies $\omega_{n}$ are defined in Eq. (8). Finally, recovering eigenfunctions $X_{k}(x)$ in Eq. (87) together with their norms in Eq. (98), we obtain:

$$
\begin{aligned}
T_{n}(t) & =\frac{F \sin \frac{(2 n+1) \pi}{2}}{\rho A L / 2} \Phi_{n}(t, \tau, 0) \\
& \left.=\frac{2 F(-1)^{n}}{\omega_{n}^{2} \rho A L}\left[\left[1-\cos \left(\omega_{n} t\right)\right)\right] H(t)-\left[1-\cos \left(\omega_{n}(t-\tau)\right)\right] H(t-\tau)\right] \\
& \left.=\frac{8 F L(-1)^{n}}{E A(2 n+1)^{2} \pi^{2}}\left[\left[1-\cos \left(\omega_{n} t\right)\right)\right] H(t)-\left[1-\cos \left(\omega_{n}(t-\tau)\right)\right] H(t-\tau)\right] .
\end{aligned}
$$

We note here that the amplitude does not depend on the density $\rho$.

2. A single impact force of zero time duration — the right side is written via the Dirac function $\delta(t)$, see Eq. (5):

$$
\begin{aligned}
T_{n}(t) & =\frac{\sin \left(\omega_{n} t\right)}{\omega_{n}} H(t) * \frac{\left\langle q(x, t) \cdot X_{n}(x)\right\rangle}{\rho A\left\|X_{k}(x)\right\|^{2}} \\
& =\frac{\sin \left(\omega_{n} t\right)}{\omega_{n}} H(t) * \delta(t) \frac{F}{\rho A\left\|X_{k}(x)\right\|^{2}}\left\langle\delta(x-L) \cdot X_{n}(x)\right\rangle \\
& =\frac{2 F(-1)^{n}}{A \sqrt{E \rho}(2 n+1) \pi} \sin \left(\omega_{n} t\right) H(t) .
\end{aligned}
$$

Both scalar product and the convolution are trivially computed because of the fundamental property of Dirac functions $F \delta(x-L)$ and $\delta(t)$.

3. A double impact problem as the first impact with an amplitude $F$ at time $t=0$ and the second impact with an amplitude $F_{1}$ at time $\tau_{1}$, see the right hand side in Eq. (6):

$$
T_{n}(t)=\frac{8 L(-1)^{n} \omega_{n}^{2}}{E A(2 n+1)^{2} \pi^{2}}\left[F \Phi_{n}(t, \tau, 0)+F_{1} \Phi_{n}\left(t, \tau, \tau_{1}\right)\right]
$$




$$
\begin{aligned}
= & \left.\frac{8 F L(-1)^{n}}{E A(2 n+1)^{2} \pi^{2}}\left[\left[1-\cos \left(\omega_{n} t\right)\right)\right] H(t)-\left[1-\cos \left(\omega_{n}(t-\tau)\right)\right] H(t-\tau)\right] \\
& +\frac{8 F_{1} L(-1)^{n}}{E A(2 n+1)^{2} \pi^{2}}\left[\left[1-\cos \left(\omega_{n}\left(t-\tau_{1}\right)\right)\right)\right] H\left(t-\tau_{1}\right) \\
& \left.-\left[1-\cos \left(\omega_{n}\left(t-\tau_{1}-\tau\right)\right)\right] H\left(t-\tau_{1}-\tau\right)\right] .
\end{aligned}
$$

Similar expressions (in square brackets) have been found by Yang et al [35] in order to formulate the cancellation conditions for the moving pulse loads represented by Delta functions for a simply supported beam.

The full solution for all three cases is then given in the form of Fourier series presented in Eq. (7) with the corresponding $T_{n}(t)$.

\subsubsection{Special selection of time $\tau_{1}$ for the double impact problem satisfying the wave cancellation condition}

Consider a case with the double impact with forces of equal amplitude $F_{1}=F$ acting in the same direction and the time after the second impact $t>\tau+\tau_{1}$. That means that the force $F$ of duration $\tau$ is applied in positive direction, and after a period of time $\tau_{1}$ the same force $F$ of duration $\tau$ is applied again. Eq. (23) is transformed with regards to the Property 1 of the response function in Eq. (11) as follows:

$$
\begin{aligned}
T_{n}(t) & =\frac{8 F L(-1)^{n} \omega_{n}^{2}}{E A(2 n+1)^{2} \pi^{2}}\left[\Phi_{n}(t, \tau, 0)+\Phi_{n}\left(t, \tau, \tau_{1}\right)\right] \\
& =\frac{32 F L(-1)^{n}}{E A(2 n+1)^{2} \pi^{2}}\left[\sin \left(\omega_{n} \frac{2 t-\tau-\tau_{1}}{2}\right) \sin \left(\frac{\omega_{n} \tau}{2}\right)\right] \cos \frac{\omega_{n} \tau_{1}}{2} .
\end{aligned}
$$

Consider Property 4 of the response functions for the double impact in the same direction. Eq. (17) for the cancellation conditions with $\omega_{n}$ in Eq. (8) is written as

$$
\alpha \frac{\pi(2 n+1)}{(2 k+1)}=\frac{\pi(2 m+1)}{2} .
$$

Now we can determine a set of values $\alpha$, satisfying Eq. (25) with $n=m$ and corresponding time of the second impact $\tau_{1}$ as follows:

1. The time $\tau_{1}$ is proportional to the first eigenperiod $P_{0}$ :

$$
k=0, \quad \alpha=\frac{1 \cdot(2 k+1)}{2}, \quad \tau_{1}=\frac{2 n+1}{2} P_{0}, \quad n=m=0,1,2 \ldots
$$

2. The time $\tau_{1}$ is proportional to the second eigenperiod $P_{1}$ :

$$
k=1, \quad \alpha=\frac{3 \cdot(2 k+1)}{2}, \quad \tau_{1}=\frac{3(2 n+1)}{2} P_{1}, \quad n=m=0,1,2 \ldots
$$

3. etc....

4. The time $\tau_{1}$ is proportional to the $(n+1)$ th eigenperiod $P_{n}$ :

$$
n, \quad \alpha=2 n+1, \quad \tau_{1}=\frac{(2 n+1)(2 k+1)}{2} P_{n}, \quad n=m=0,1,2 \ldots
$$

Thus, according to Eq. (26), the second counter impact should be applied at the time $\tau_{1}$ which is equal to uneven number of first eigenperiods $P_{0}$ divided by 2 . This gives the wave cancellation condition for the considered double impact in the same direction.

We consider this effect comparing the single and double impact problem in the following numerical example. 
2.4 Numerical example of the double impact problem in the same direction

The numerical solution is exemplary computed for the following parameters: $L=50, E=2.1 \times 10^{11} \mathrm{~N} \mathrm{~m}^{-2}$, $A=1 \mathrm{~m}^{2}, \rho=7800 \mathrm{~kg} \mathrm{~m}^{-3}$, the force $P=100,000 \mathrm{~N}$ is applied for a duration $\tau=0.2 P_{0}$ and the counter impact is applied at time $\tau_{1}=\frac{5}{2} P_{0}$ (uneven number of the first eigen period $T_{0}$ divided by 2). In this case the first eigenperiod is $P_{0}=3.854 \times 10^{-2} \mathrm{~s}, \tau=7.708 \times 10^{-3} \mathrm{~s}$ and the time of application of the counter impact is $\tau_{1}=9.636 \times 10^{-2} \mathrm{~s}$. The computation of displacement at the point $x=L$ is shown for the single impact in Fig. 2, and for the double impact in Fig. 3. One can see, that the counter-impact leading to the wave cancellation is recognized after the time $\tau_{1}=\frac{5}{2} P_{0}$ when the vibrations are fully disappeared. It should be noted, that displacements as well as velocities are zero at each point $x \in[0, L]$. Thus, the energy supplied with the first impact is canceled with the counter impact. In both cases, the number of terms for the Fourier series is taken as $n=100$.

\section{Wave cancellation conditions for the double impact problem in an arbitrary structure}

The double impact problem and corresponding wave cancellation conditions, studied in Sect. 2 for 1D bar, can be generalized for any arbitrary model formulated in linear continuum mechanics. Consider an impact problem within the linear mechanics in a general form. The linear statical problem for any type of modeled structures (bar, beam, shell, continuum) can be described employing the corresponding linear semi-elliptic operator $L u$ in the following form

$$
L u(x)+q(x)=0, \quad x \in \Omega,
$$

where $u(x)$ is a generalized degree of freedom vector. This vector, depending on the mechanical model, may contain not only displacements components in the corresponding 1D, 2D or 3D space as for conventional models in continuum mechanics, but also angular degree of freedoms as for models in various beam and shell theories, pressure as for acoustics models etc. etc. In this case, $q(x)$ is a generalized external load vector, corresponding to those degrees of freedom. Equation (29) is describing equilibrium conditions of the structure occupied in the domain $\Omega$. Domain $\Omega$ is, in due course, a geometrical finite domain - a 3D object, a surface or a curve - the last depends on the mechanical model. Both Neumann and Dirichlet boundary conditions acting on the boundaries $\partial \Omega_{\mathrm{N}}$ and $\partial \Omega_{\mathrm{D}}$ are considered in the linear form with the corresponding linear operators $B_{\mathrm{N}}$ and $B_{\mathrm{D}}$ as

$$
B_{\mathrm{N}} u(x)=b_{\mathrm{N}}, \quad x \in \partial \Omega_{\mathrm{N}},
$$

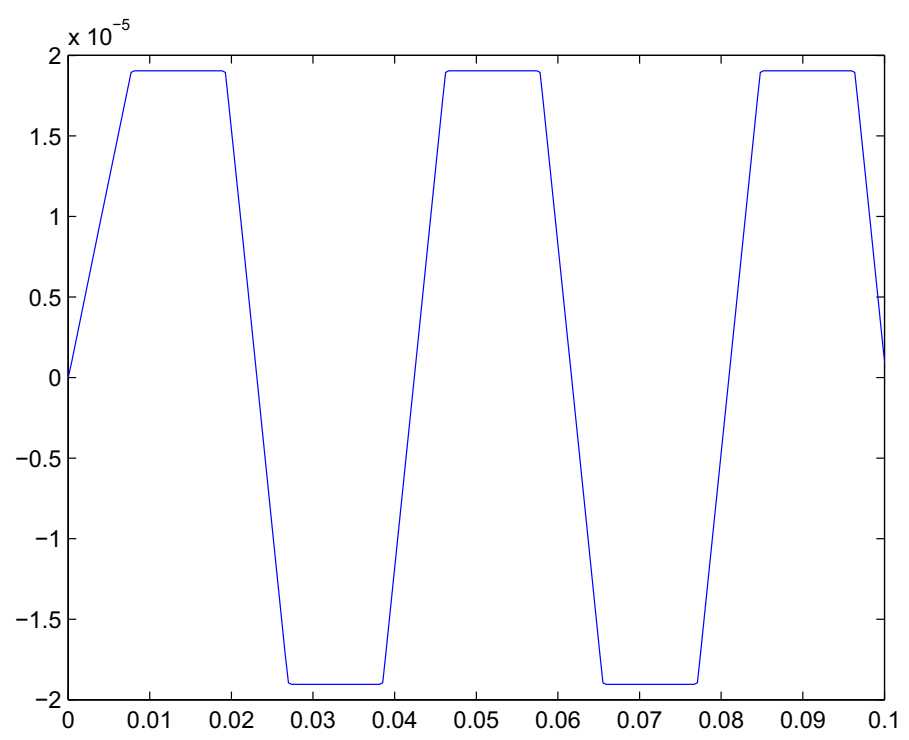

Fig. 2 Single impact of duration $\tau=7.708 \times 10^{-3}$. Displacement of the bar at the point $x=L$ versus time 


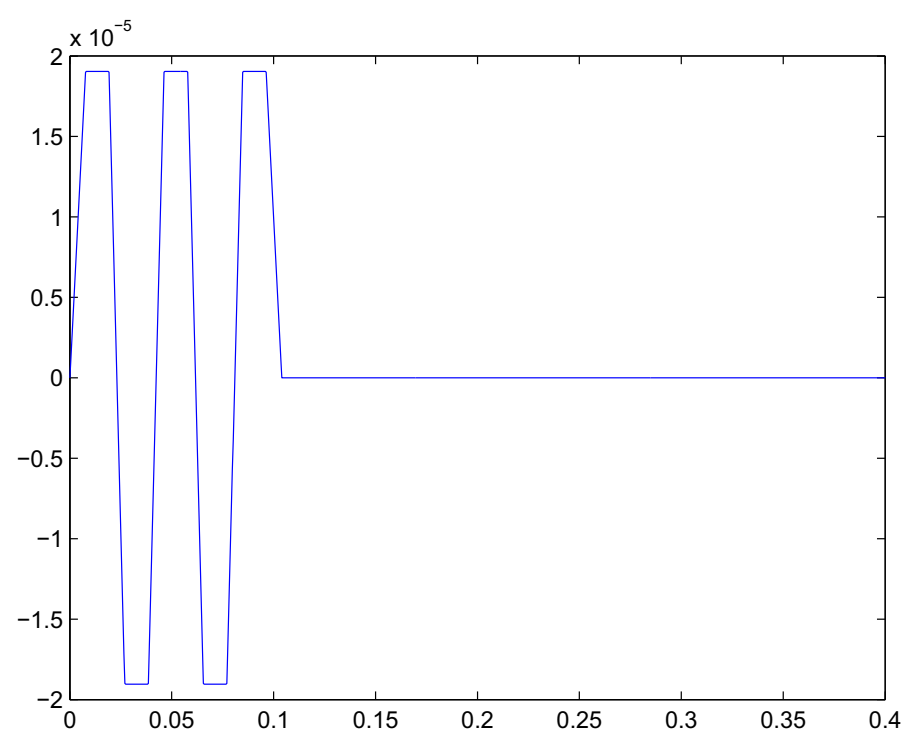

Fig. 3 Double impact of duration $\tau$ : counter impact is applied at $\tau_{1}=9.636 \times 10^{-2}$. Displacement of the bar at the point $x=L$ versus time. The wave is fully canceled after application of the second impact

$$
B_{\mathrm{D}} u(x)=b_{\mathrm{D}}, \quad x \in \partial \Omega_{\mathrm{D}}
$$

The non-homogeneous Neumann boundary conditions Eq. (30) can be included in the external force $q(x)$ using generalized functions acting on the corresponding boundary $\partial \Omega_{\mathrm{N}}$. These generalized functions depending on the geometry of the domain $\Omega$ are represented in due course by the Dirac vector function $\delta\left(x-x_{0}\right)$ acting at the point $x_{0} \in \mathfrak{R}$, or on a curve or on a surface.

The linear dynamic problem for the structure with a constant density $\rho$ is formulated in the form:

$$
\rho \frac{\partial^{2} u}{\partial t^{2}}=L u(x)+q(x, t), \quad x \in \Omega .
$$

The structure is in idle state at the beginning, therefore, initial conditions for the impact problem are considered homogeneous:

$$
\left.\frac{\partial u}{\partial t}\right|_{t=0}=0,\left.\quad u\right|_{t=0}=0
$$

The external force $q(x, t)$, representing the double impact problem acting at the point $x=a \in \mathfrak{R}$ is written similar to Eq. (6) via the Dirac vector function

$$
q_{\left(F F_{1}\right)}(x, t)=F \delta(x-a)[H(t)-H(t-\tau)]+F_{1} \delta(x-a)\left[H\left(t-\tau_{1}\right)-H\left(t-\tau-\tau_{1}\right)\right],
$$

Following the solution of the dynamics problem in Eq. (32) via the Fourier series and convolution, we obtain the corresponding eigenvalue problem as:

$$
L X+\lambda X=0
$$

with the corresponding homogeneous boundary conditions

$$
\begin{aligned}
& B_{\mathrm{N}} X(x)=0, \quad x \in \partial \Omega_{\mathrm{N}}, \\
& B_{\mathrm{D}} X(x)=0, \quad x \in \partial \Omega_{\mathrm{D}} .
\end{aligned}
$$

Further on, we assume, without the loss of generality, that the Dirichlet boundary condition is homogeneous. This generalization is, however, valid for stationary boundary condition, i.e. in Eq. (31) $b_{\mathrm{N}}$ should not depend on the time $t$. As is known [33], the eigenvalue problem with a positive linear elliptic operator has the solution in the form of positive eigenvalues $\lambda_{n}, n=1,2, \ldots$ and orthogonal eigenfunctions $X_{n}(x), n=1,2, \ldots$ We 
will consider further a case with non-zero eigenvalues-this means that the corresponding structure is fixed properly excluding rigid body degree of freedoms.

The solution of Eq. (32) following the Fourier method is searched in the form

$$
u(x, t)=\sum_{n} \ddot{T}_{n}(t) X_{n}(x)
$$

Using the linearity of the operator $L u$ and the corresponding eigenvalue problem (35)-(37) the dynamical equation (32) is written as

$$
\sum_{n} \rho \ddot{T}_{n}(t) X_{n}(x)+\sum_{n} \lambda_{n} T_{n}(t) X_{n}(x) .=q(x, t) .
$$

Using then the orthogonality of the eigenfunctions $X_{n}$ and performing the scalar product with the eigenfunction $X_{n}$ in the corresponding Hilbert space, Eq. (39) is transformed into a set of ordinary differential equations

$$
\ddot{T}_{n}(t)+\omega_{n}^{2} T_{n}(t)=\frac{\left\langle q(x, t) \cdot X_{n}(x)\right\rangle}{\rho\left\|X_{n}(x)\right\|^{2}},
$$

where the scalar product is defined in the corresponding Hilbert space in the domain $\Omega$ :

$$
\left\langle q(x, t) \cdot X_{n}(x)\right\rangle=\int_{\Omega} q(x, t) X_{n}(x) \mathrm{d} x .
$$

The norm $\left\|X_{n}(x)\right\|$ is then constructed with respect to this scalar product. The eigenfrequency is denoted as

$$
\omega_{n}=\sqrt{\frac{\lambda_{n}}{\rho}}
$$

Each of Eqs. (40) should satisfy the initial conditions arising from the integration of the initial conditions in Eq. (33):

$$
T_{n}(0)=0: \text { no initial displacements, } \frac{\mathrm{d} T_{\mathrm{n}}}{\mathrm{d} t}(0)=0: \text { no initial velocity. }
$$

Application of the Lemma 1 in Appendix A gives us the following solution of Eq. (40) in the form of a convolution:

$$
T_{n}(t)=\frac{\sin \omega_{n}}{\omega_{n}} * \frac{\left\langle q(x, t) \cdot X_{n}(x)\right\rangle}{\rho\left\|X_{n}(x)\right\|^{2}}
$$

Now all properties of the response function are necessary in order to determine the time $\tau_{1}$ of application of the second counter impact leading to the wave cancellation. For an arbitrary structure this is enforced by the second impact in which the application time is chosen to be proportional to the $k$ th eigen period $P_{k}$ fully similar to the 1-D example, see Eq. (15):

$$
\tau_{1}=\alpha P_{k}
$$

By specific selection of the time $\tau_{1}$, it is possible to construct the wave cancellation conditions for both cases: for the impact in the same direction as well as for the impact in the opposite direction. 


\subsection{Sum of two impacts in the same directions: wave cancellation conditions}

The first impact force $F$ of duration $\tau$ is applied at the point $x=a$. The corresponding functions $T_{n}^{\text {first }}(t)$ are calculated using Eq. (44) and the response function in Eq. (10):

$$
T_{n}^{\text {first }}(t)=\frac{P X_{n}(a)}{\rho\left\|X_{n}(x)\right\|^{2}} \Phi_{n}(t, \tau, 0) .
$$

The second impact is considered in the same direction and is applied at time $\tau_{k}$. All terms of the Fourier series after the time $\tau_{k}: \tau_{k+1}, \ldots, \tau_{n}, \ldots$ are taken into account:

$$
T_{n}^{\text {second }}(t)=\frac{P X_{n}(a)}{\rho\left\|X_{n}(x)\right\|^{2}} \Phi_{n}\left(t, \tau, \tau_{k}\right) .
$$

According to the first property of the response function in Eq. (11) the sum of the functions after the time $t>\tau+\tau_{k}$ is written as

$$
\begin{aligned}
T_{n}(t) & =T_{n}^{\mathrm{first}}(t)+T_{n}^{\mathrm{second}}(t) \\
& =\frac{4 P X_{n}(a)}{\rho\left\|X_{n}(x)\right\|^{2} \omega_{n}^{2}}\left[\sin \left(\omega_{n} \frac{2 t-\tau-\tau_{k}}{2}\right) \sin \left(\frac{\omega_{n} \tau}{2}\right)\right] \cos \frac{\omega_{n} \tau_{k}}{2} .
\end{aligned}
$$

Displacements of the structure for the second impact are represented not only with a single term as in the previous particular 1D example, but also with all terms including times $\tau_{k}, \tau_{k+1}, \ldots$ In order to recover zeroes of the response function it is required from Eq. (17) that $\alpha \frac{\omega_{n}}{\omega_{k}}=\frac{2 m+1}{2}$. This can be fulfilled as a case of a double counter impact with $\omega_{k}=\omega_{n}$ and for $\alpha=\frac{2 m+1}{2}$. Thus, application of the second impact at the time equals to the uneven number of kth eigen period divided by $2: \tau_{m}=\frac{2 m+1}{2} \tau_{k}$ leads to the wave cancellation condition and to the elimination of the displacements in the whole structure leads to the wave cancellation condition and to the elimination of the displacements in the whole structure.

\subsection{Sum of two impacts in the opposite directions: wave cancellation conditions}

The first impact force $F$ of duration $\tau$ is applied at the point $x=a$ and is represented by Eq. (46). The second impact is considered in the opposite direction and is represented as

$$
T_{n}^{\text {second }}(t)=\frac{-P X_{n}(a)}{\rho\left\|X_{n}(x)\right\|^{2}} \Phi_{n}\left(t, \tau, \tau_{k}\right) .
$$

According to the 2-st property of the response function in Eq. (12) the sum of the functions after the time $t>\tau+\tau_{k}$ is written as:

$$
\begin{aligned}
T_{n}(t) & =T_{n}^{\text {first }}(t)+T_{n}^{\text {second }}(t) \\
& =\frac{4 P X_{n}(a)}{\rho\left\|X_{n}(x)\right\|^{2} \omega_{n}^{2}}\left[\cos \left(\omega_{n} \frac{4 t-\tau-2 \tau_{k}}{4}\right) \sin \left(\frac{\omega_{n} \tau}{2}\right)\right] \sin \frac{\omega_{n} \tau_{k}}{2} .
\end{aligned}
$$

In order to recover zero of the response function it is required from Eq. (19) that $\alpha \frac{\omega_{n}}{\omega_{k}}=m$. This can be fulfilled as a case of a double counter impact with $\omega_{k}=\omega_{n}$ and for $\alpha=m$. Thus, application of the second impact at the time equals to the integer number of $m$ of the $k$ th eigenperiod $\tau_{k}: \tau_{m}=m \tau_{k}$ leads to the elimination of the displacements.

Remark 2 (On resonance conditions) If, in the definition of the wave cancellation conditions for both same and opposite directions, the direction of a force is changed into opposite, then the resonance condition is derived. In the case of a double impact it leads to the doubling of an amplitude after the second impact.

Within the example from Sect. 2, a series impacts sequentially in times $P_{1}, P_{2}, \ldots, P_{m}, \ldots$ will lead to the resonance phenomena as doubling of an amplitude during each impact. Conditions for the eigenfrequencies similar to Eq. (25) have been found by Lu et al. [22] for the cancellation and resonance conditions caused by the equidistant moving pulse loads in a pile-supported viaduct. 


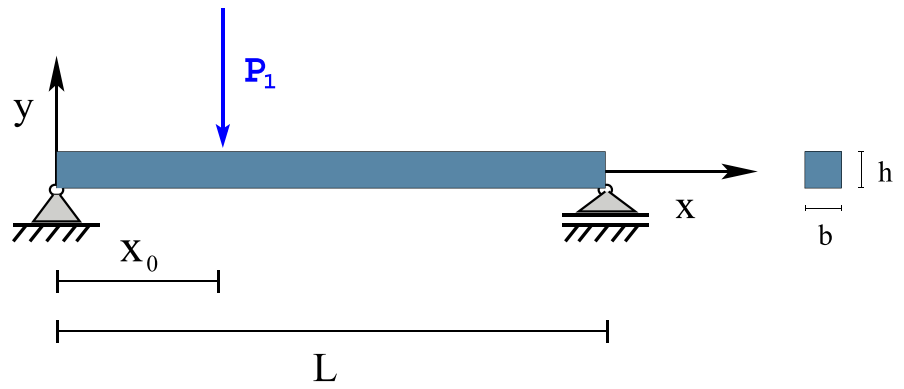

Fig. 4 Beam loaded with an impact $P$ of duration $\tau$ at the distance $x_{0}$

\section{Solution of the double impact problem for the simply supported Bernoulli beam}

As an example of the developed strategy in Sect. 3 we are showing here the derivation of the cancellation condition for the simply support beam exemplarily for the double impact in the opposite direction. Consider a beam with length $l$, constant cross-section $A$ and second moment of area $I$, made of elastic material with elasticity modulus $E$ and constant density $\rho$. The beam is simply supported, see Fig. 4. At time $t=0$ the constant impacting load $F$ of duration $\tau$ is applied at the distance $x_{0}$. The dynamic motion is described by the vertical displacement $u(x, t)$, which according to the Bernoulli beam hypothesis should satisfy to the following PDE, see e.g. in [13] and other solutions in [5]:

$$
E I \frac{\partial^{4} u}{\partial x^{4}}+\rho A \frac{\partial^{2} u}{\partial t^{2}}=q(x, t) .
$$

Here the distributed force $q(x, t)$ (physical dimension $\left[\mathrm{N} \mathrm{m}^{-1}\right]$ ) is represented via the Dirac function as $q(x, t)=F(t) \delta\left(x-x_{0}\right)$. The function $F(t)$, depending on the type of impact, is represented similar to Eqs. $(4,5,6)$.

The operator $L u$ with regards to Eq. (32) is now represented as follows:

$$
L u \equiv-\frac{E I}{\rho} \frac{\partial^{4} u}{\partial x^{4}} .
$$

The function $u(x, t)$ for the simple support should satisfy the following boundary conditions:

- zero displacements on supports (Dirichlet boundary conditions):

$$
u(0, t)=0, u(l, t)=0, \quad \forall t>0 ;
$$

- zero moments on supports (Neumann boundary conditions)

$$
M(0, t)=0, M(l, t)=0 \Rightarrow \frac{\partial^{2} u}{\partial x^{2}}(0, t)=0, \frac{\partial^{2} u}{\partial x^{2}}(l, t)=0, \quad \forall t>0 ;
$$

and the following initial conditions (assuming no motion at the beginning):

$$
u(x, 0)=0,\left.\frac{\partial u}{\partial t}\right|_{t=0}=0 \text { for } x \in[0, l] .
$$

In order to obtain the solution in the form of Fourier series, following the strategy shown generally in Sect. 3, we have to solve the corresponding eigenvalue problem for $X_{n}(x)$, and then obtain the solution in the form of convolution for $T_{n}(t)$.

4.1 The corresponding eigenvalue problem and differential equation for $T_{n}(t)$

The eigenvalue problem for the corresponding operator $L u$ in Eq. (52) is formulated from the general statement in Eq. (35), in which $\lambda^{4}$ is taken as eigenvalue for further convenience, 


$$
X^{(4)}-\lambda^{4} X=0 .
$$

The corresponding boundary conditions (generally derived also from Eqs. (36)-(37)) —zero displacements (from Eq. (53)) and zero bending moments (from Eq. (54)) at both ends of the beam-are written as follows:

$$
X(0)=0, X(l)=0, \quad X^{\prime \prime}(0)=0, X^{\prime \prime}(l)=0 .
$$

This standard solution is available from many books on dynamics and vibration theory. The corresponding eigenvalues and eigenfunctions are written as

$$
\begin{aligned}
\lambda_{n} & =\frac{\pi n}{l}, \quad n=1,2,3, \ldots, \\
X_{n}(x) & =\sin \left(\frac{\pi n}{l} x\right), \quad n=1,2,3, \ldots,
\end{aligned}
$$

and the square of the norm for eigenfunctions is calculated as

$$
\left\|X_{n}(x)\right\|^{2}=\int_{0}^{l} \sin ^{2}\left(\frac{\pi n}{l} x\right) \mathrm{d} x=\frac{l}{2} .
$$

The differential equation (40) for the time variable $T_{n}(t)$ in this case is written as:

$$
\ddot{T}_{n}(t)+\omega_{n}^{2} T_{n}(t)=\frac{\int_{0}^{l} q(x, t) X_{n}(x) \mathrm{d} x}{\rho A\left\|X_{n}(x)\right\|^{2}},
$$

where the corresponding eigen frequencies $\omega_{n}$ for the beam are defined as:

$$
\omega_{n}^{2}=\frac{E I \lambda_{n}^{4}}{\rho A} \Longrightarrow \omega_{n}=\sqrt{\frac{E I}{\rho A}}\left(\frac{\pi n}{l}\right)^{2} .
$$

4.2 Numerical example: wave cancellation condition for the double impact problem in the opposite direction

The double impact for the beam problem is formulated as a composition of two impacts with the same duration $\tau$ applied at the same position $x=x_{1}$. The first impact $F_{1}$ is applied at time $t=0$, the second impact $F_{2}$ is applied at time $t=\tau_{1}$. In this case, the external force $q_{\left(F F_{1}\right)}(x, t)$ in Eq. (34) is formulated for the point at the mid-line of a beam $a=x_{1}$ :

$$
q_{\left(F F_{1}\right)}(x, t)=F_{1} \delta\left(x-x_{1}\right)(H(t)-H(t-\tau))+F_{2} \delta\left(x-x_{1}\right)\left(H\left(t-\tau_{1}\right)-H\left(t-\tau_{1}-\tau\right)\right) .
$$

Now, using definition of the response function 2 we can write the solution for the time variable $T_{n}(t)$ after all transformations in the following form:

$$
\begin{aligned}
T_{n}(t) & =T_{f} * F(x, t) \\
& =\frac{2}{\rho l A}\left[F_{1} X_{n}\left(x_{1}\right) \Phi(t, \tau, 0)+F_{2} X_{n}\left(x_{1}\right) \Phi\left(t, \tau, \tau_{1}\right)\right] .
\end{aligned}
$$

Let us study numerically the case with the double impact in opposite direction with the force $F=F_{1}=-F_{2}$. The full solution of the problem is given by the following Fourier series:

$$
\begin{aligned}
u(x, t)= & \sum_{n=1}^{\infty} \frac{2 F}{l A \rho \omega_{n}^{2}}\left[\left(\left(\cos \left(\omega_{n}(t)\right)-1\right) H(t)\right.\right. \\
& \left.-\left(\cos \left(\omega_{n}(t-\tau)\right)-1\right) H(t-\tau)\right] \sin \left(\frac{\pi n}{l} x_{0}\right) \sin \left(\frac{\pi n}{l} x\right)
\end{aligned}
$$




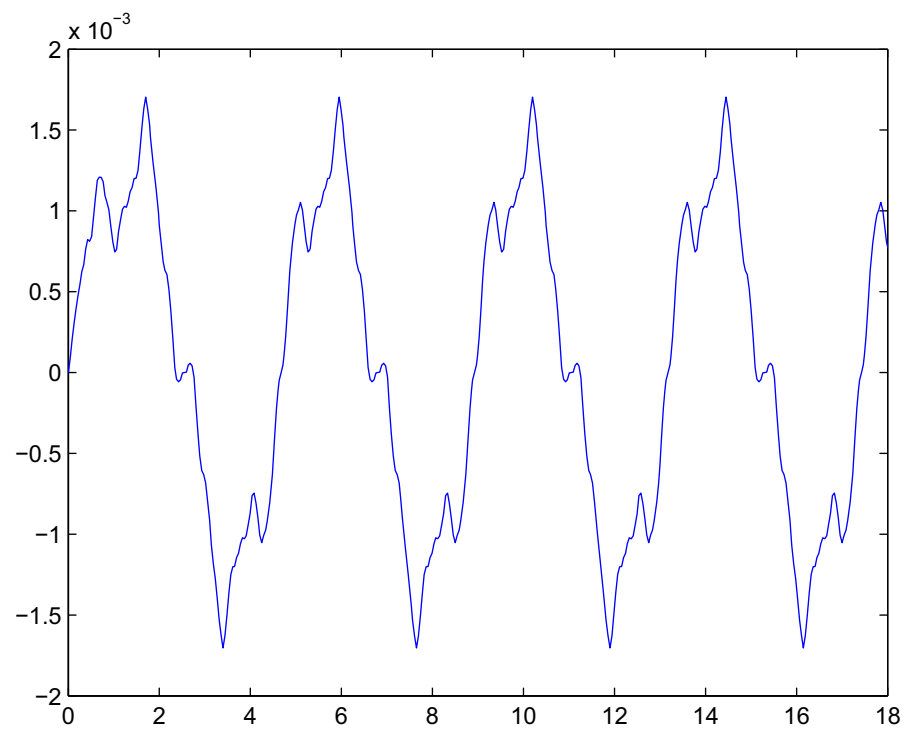

Fig. 5 Vertical displacement $u$ at the point $x=x_{0}$ of applied force $F$. Single impact

$$
\begin{aligned}
& -\sum_{n=1}^{\infty} \frac{2 F}{l A \rho \omega_{n}^{2}}\left[\left(\left(\cos \left(\omega_{n}\left(t-\tau_{1}\right)-1\right) H\left(t-\tau_{1}\right)\right.\right.\right. \\
& \left.-\left(\cos \left(\omega_{n}\left(t-\tau-\tau_{1}\right)\right)-1\right) H\left(t-\tau-\tau_{1}\right)\right] \sin \left(\frac{\pi n}{l} x_{0}\right) \sin \left(\frac{\pi n}{l} x\right) .
\end{aligned}
$$

The condition for zeros of the response function Property 5 in Eq. (19) and also the wave cancellation condition for the double impact in opposite direction in Sect. 3.2 with regards to the eigen frequencies $\omega_{n}$ in Eq. (61) leads to the following condition:

$$
\alpha \frac{n^{2}}{k^{2}}=m
$$

which can be resolve in integer numbers as $n=k, \alpha=k$. Thus, the time of application of the second impact in the opposite direction satisfying the wave cancellation condition is then proportional to integer number of the first period $P_{1}$.

The following data for the numerical example computation are taken: elasticity modulus $E=2.1 \times$ $10^{11} \mathrm{~N} \mathrm{~m}^{2}$, density $\rho=7800 \mathrm{~kg} \mathrm{~m}^{-3}$, length $L=100 \mathrm{~m}$, applied force $F=10,000 \mathrm{~N}$, moment of inertia $I=\frac{1}{12} \mathrm{~m}^{4}$, area $A=1 \mathrm{~m}^{2}$, position of the applied impacting force $x_{0}=10 \mathrm{~m}$. The duration of the impact $\tau=0.2 \cdot P_{1}$, time of the application of the second impact $\tau_{1}=3 \cdot P_{1}$. The fist period $P_{1}$ is computed via the the first eigenfrequency

$$
\frac{2 \pi}{P_{1}}=\omega_{1}=\sqrt{\frac{E I}{\rho}}\left(\frac{\pi}{l}\right)^{2} .
$$

Results of computations for the single impact are shown in Fig. 5 and for the double impact illustrating the wave cancellation in Fig. 6. The vibration is fully canceled after the third period as expected.

\section{Double impact on the cantilever beam: analysis for both same and opposite directions}

As an example with arbitrary eigenvalues we consider another boundary conditions for the beam modeled considered in Sect. 4: the double impact at the free end on the cantilever beam with length $l$. This simple case representing the arbitrary structure and is not allowing to construct special cases such as for 1D bar and simply supported beam, because, solutions of the characteristic equation for eigenvalues have no closed form and 


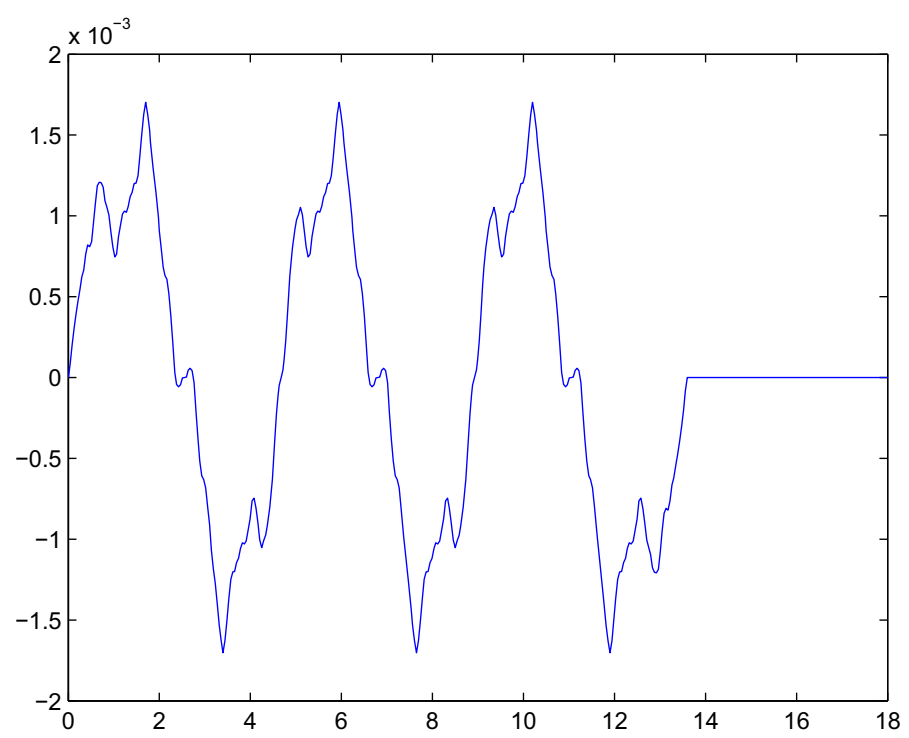

Fig. 6 Vertical displacement $u$ at the point $x=x_{0}$ of applied force $F$. Double impact and wave cancellation after the third period

should be found numerically. The operator $L u$ remains as in Sect. 4, see Eq. (52). Further necessary operations are as follows (can be found also from the monographs, see e.g. [13]):

1. Equation for definition of the eigenvalues $\lambda_{n}$-the characteristic equation:

$$
\sinh \left(\lambda_{n} l\right) \cos \left(\lambda_{n} l\right)=-1
$$

The solution can be obtained numerically-in our case $\lambda_{1} l=1.875, \lambda_{2} l=4.694 \ldots$

2. Corresponding eigenfunctions $X_{n}(x)$ :

$$
\begin{aligned}
X_{n}(x)= & \left(\cos \left(\lambda_{n} l\right)+\cosh \left(\lambda_{n} l\right)\right)\left(\cos \left(\lambda_{n} x\right)-\cosh \left(\lambda_{n} x\right)\right) \\
& +\left(\sin \left(\lambda_{n} l\right)-\sinh \left(\lambda_{n} l\right)\right)\left(\sin \left(\lambda_{n} x\right)-\sinh \left(\lambda_{n} x\right)\right) .
\end{aligned}
$$

3. Computation of the norm $\left\|X_{n}(x)\right\|$.

The lengthy expression can be computed in the closed form in any mathematical software (MATLAB etc.) and is not given here.

The cantilever beam with the same material parameters as in the previous example: elasticity modulus $E=2.1 \times 10^{11} \mathrm{~N} \mathrm{~m}^{2}$, density $\rho=7800 \mathrm{~kg} \mathrm{~m}^{3}$, length $L=100 \mathrm{~m}$, applied force $P=10,000 \mathrm{~N}$, moment of inertia $I=\frac{1}{12} \mathrm{~m}^{4}$, area $A=1 \mathrm{~m}^{2}$ is subjected to the first impact at the end $x_{0}=100 \mathrm{~m}$. The duration of the impact $\tau=0.2 P_{1}=2.39 \mathrm{~s}$.

Two cases for the illustration of double impact results in both same and opposite directions, discussed in Sects. 3.1 and 3.2, are considered. In addition, the influence of the number of terms in the Fourier series is studied.

\subsection{Wave cancellation conditions: double impact in the opposite direction}

The single impact is forcing the cantilever beam to vibrate. The displacement at the end of the beam is shown in Fig. 7-the second impact is not applied here. Then the time of the first application time for the second impact is chosen as $\tau_{1}=4 P_{1}=4 \times 11.93=47.72 \mathrm{~s}$, where the fist period $P_{1}$ is computed via the first eigenfrequency. Other necessary terms for the full Fourier series are $\tau_{2}=3 P_{2}=3 \times 1.90 \mathrm{~s}, \tau_{3}=3 \times P_{3}=3 \times 0.68 \mathrm{~s}, \ldots$. The result is shown in Fig. 8. In order to study the influence of the high frequency terms, the second impact is computed with only one term $\left(\tau_{1}\right)$ and with two terms $\left(\tau_{1}, \tau_{2}\right)$ of the Fourier series and shown for the region near $\tau_{1}$ in Fig. 9. It can be seen that the contribution of the terms higher than 2 is rather negligible. 


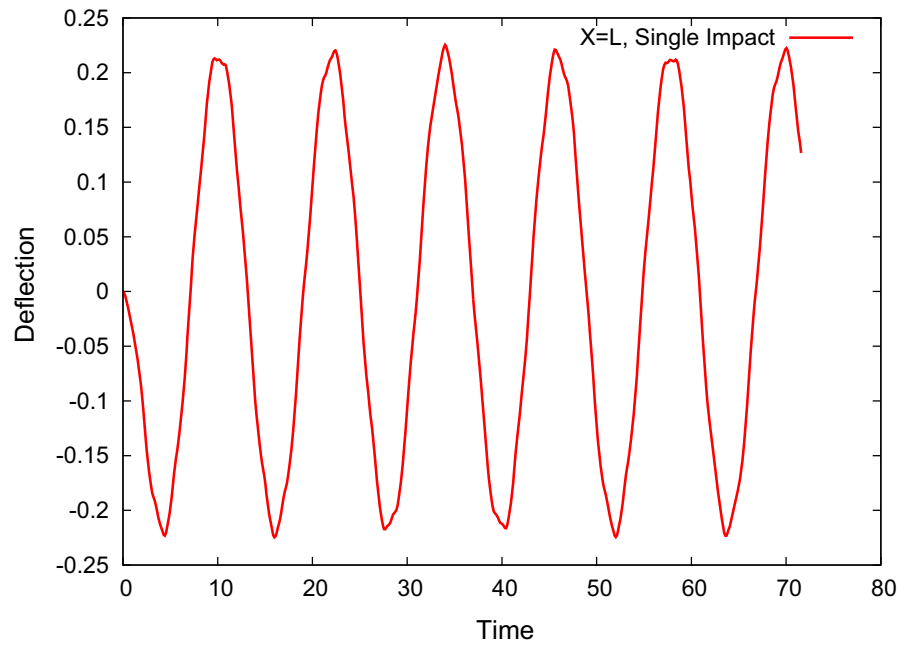

Fig. 7 Vertical displacement $u$ at the point $x=l$ of applied force $P$. Single impact

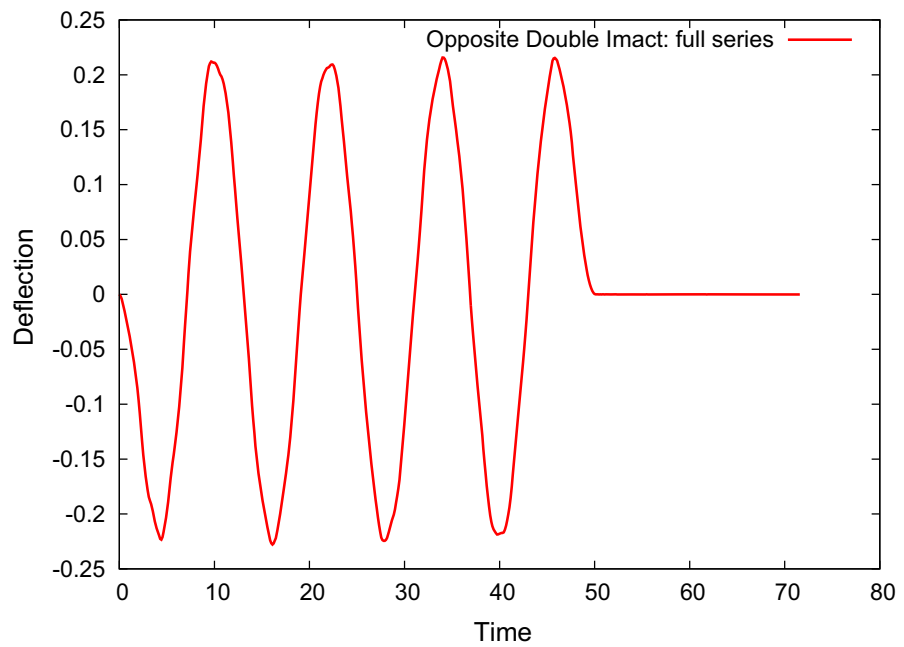

Fig. 8 Vertical displacement $u$ at the point $x=l$ of applied force $P$. The double opposite impact. Full Fourie series

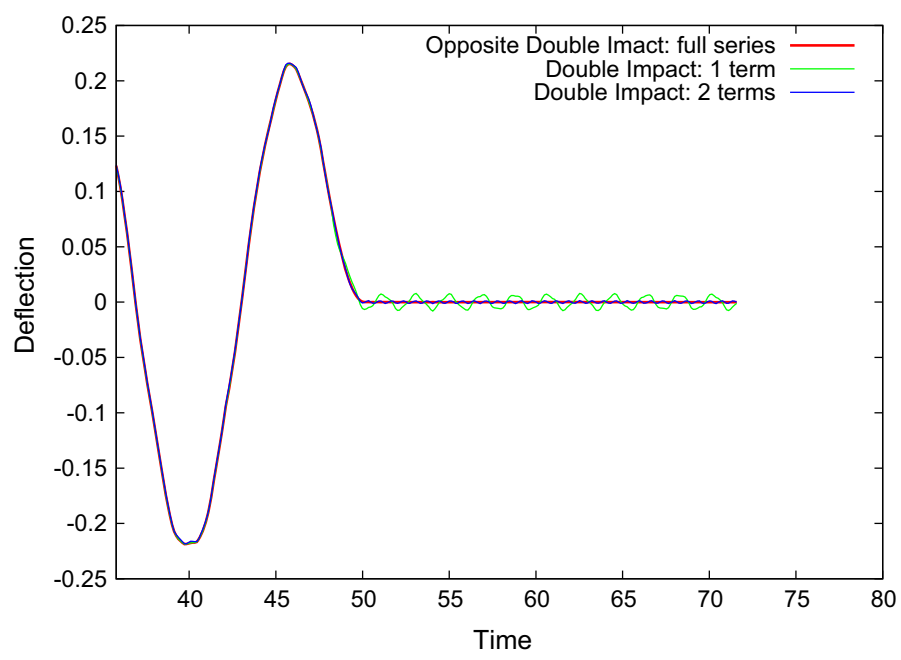

Fig. 9 Vertical displacement $u$ at the point $x=l$ of applied force $P$. The double opposite impact leading to the wave cancellation. Influence of the number of terms in Fourier series 


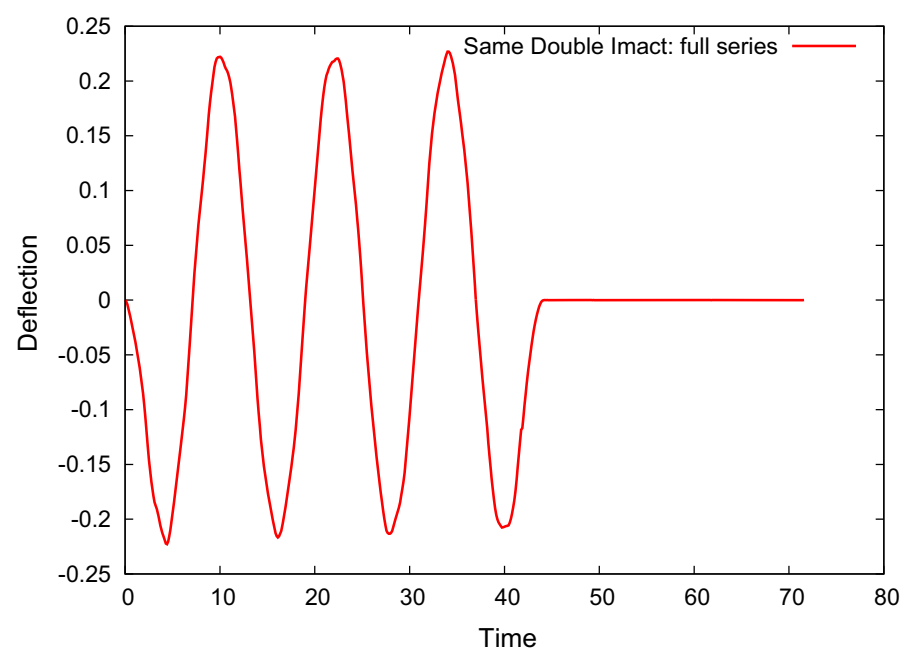

Fig. 10 Vertical displacement $u$ at the point $x=l$ of applied force $P$. Double impact in the same direction leading to the wave cancellation. Full Fourier series

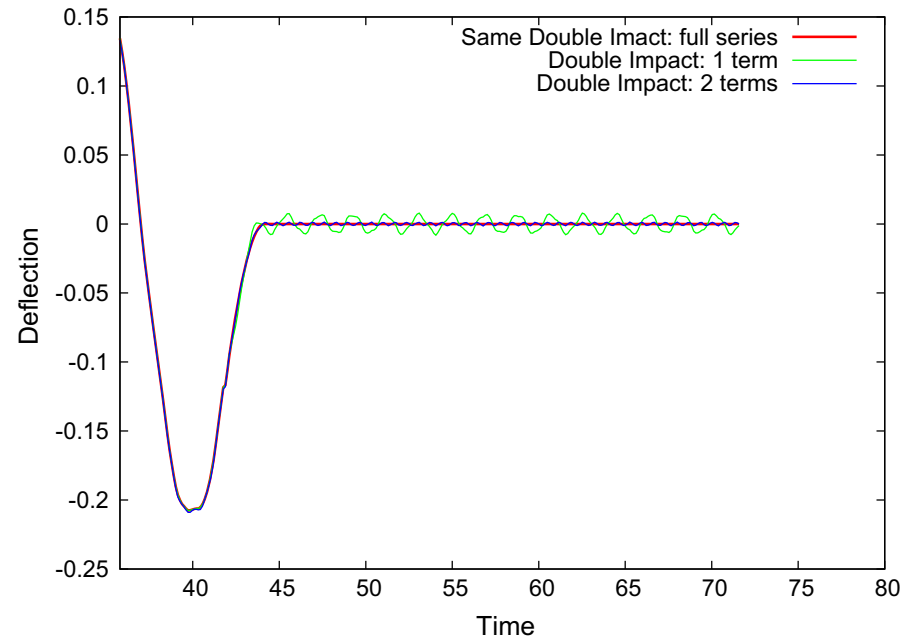

Fig. 11 Vertical displacement $u$ at the point $x=l$ of applied force $P$. Wave cancellation. Influence of the number of terms in Fourier series

\subsubsection{Wave cancellation conditions: double impact in the same direction}

Now, the opposite impact is applied at uneven divided by 2 number of the first eigenperiod $\tau_{1}=3.5 T_{1}=$ $41.76 \mathrm{~s}$, see displacement at the end of the beam in Fig. 10. Again for the comparison the second impact is computed with only one term $\left(\tau_{1}\right)$ and with two terms $\left(\tau_{1}, \tau_{2}\right)$ of the Fourier series and shown for the region nearby $\tau_{1}$ in Fig. 11. It can be seen that the contribution of terms higher than 2 is rather negligible.

The displacements are fully canceled after the time $t=\tau_{1}+\tau$ for both cases with the same and the opposite double impacts, i.e. during the duration time of the second impact $\tau$ after the application time $\tau_{1}$.

Remark 3 (Structure of the second counter impact) It should be noted that in the case of an arbitrary structure the wave cancellation conditions for the double impact in both cases require the second impact structure in terms of response functions $\Phi_{n}\left(t, \tau, \tau_{n}\right)$ (see Eqs. (47) and (49)) activated consequently in sequence of times $\tau_{1}, \tau_{2}, \ldots$. Only simple structure of eigenvalues in previous Sects. 2.3.1 and 4.2 allows to define a single value $\tau_{1}$ for all terms. However, as shown in numerical examples Figs. 9 and 11 the wave is dominantly canceled even within the first term, and almost fully cancelled if only terms are taken into account. 


\section{Double impact on the membrane: numerical example for the opposite direction}

As an example with 2D inner geometry, we consider a rectangular membrane in a plane with size $a \times b$, with thickness $h$, made of material with constant density $\rho$. The membrane is under uniform pretension stress $N$ (physical dimension $\left[\mathrm{N} \mathrm{m}^{-1}\right]$ ). At the time $t=0$ the constant impacting load $F$ of duration $\tau$ is applied at the point $\left(x_{0}, y_{0}\right)$. The dynamic motion is described by a vertical displacement function $w(x, y, t)$, which is satisfying the following equation, see e.g. in [13]:

$$
\rho h \frac{\partial^{2} w}{\partial t^{2}}-N \Delta w=q(x, y, t), \quad \text { with Laplacian } \triangle() \equiv \frac{\partial^{2}()}{\partial x^{2}}+\frac{\partial^{2}()}{\partial y^{2}} .
$$

The distributed force $q(x, y, t)$ with physical dimension $\left[\mathrm{N} \mathrm{m}^{-2}\right]$ is represented in this case via the Dirac function on the plane $\delta\left(x-x_{0}\right) \delta\left(y-y_{0}\right)$ (namely $a=\left(x_{0}, y_{0}\right)$ and $F=P, F_{1}=-P$ in Eq. (34) for the arbitrary structure) as follows:

$$
\begin{aligned}
q_{(P,-P)}(x, y, t)= & P \delta\left(x-x_{0}\right) \delta\left(y-y_{0}\right)[H(t)-H(t-\tau)] \\
& -P \delta\left(x-x_{0}\right) \delta\left(y-y_{0}\right)\left[H\left(t-\tau_{1}\right)-H\left(t-\tau-\tau_{1}\right)\right] .
\end{aligned}
$$

The membrane is fixed at all boundary lines:

$$
w(0, y, t)=0, w(a, y, t)=0, w(x, 0, t)=0, w(x, b, t)=0 .
$$

The operator $L w$ with regards to Eq. (32) is represented so far as follows:

$$
L w \equiv \frac{N}{h} \Delta w=\frac{N}{h}\left[\frac{\partial^{2} w}{\partial x^{2}}+\frac{\partial^{2} w}{\partial y^{2}}\right] .
$$

The eigenvalue problem for this operator $L w$ is formulated from the general statement in Eq. (35), in which $\lambda_{n, m}^{2}$ is taken as eigenvalue for further convenience,

$$
\left[\frac{\partial^{2} X}{\partial x^{2}}+\frac{\partial^{2} X}{\partial y^{2}}\right]+\lambda^{2} X=0 .
$$

Eigenfunction $X$ should satisfy the following boundary conditions (generally derived also from Eq. (37)): zero displacements (from Eq. (71)) as Dirichlet boundary conditions.

The solution of this problem (can be also found in literature for the vibration theory, see e.g. in [13]) is represented as follows:

$$
\begin{aligned}
\lambda_{n, m}^{2} & =\left(\frac{\pi n}{a}\right)^{2}+\left(\frac{\pi m}{b}\right)^{2}, \quad n, m=1,2,3, \ldots, \\
X_{n, m}(x, y) & =\sin \left(\frac{\pi n}{a} x\right) \sin \left(\frac{\pi m}{b} y\right), \quad n, m=1,2,3, \ldots,
\end{aligned}
$$

and the square of the norm for eigenfunctions is calculated as

$$
\left\|X_{n, m}(x)\right\|^{2}=\int_{0}^{a} \int_{0}^{b} \sin ^{2}\left(\frac{\pi n}{a} x\right) \sin ^{2}\left(\frac{\pi m}{b} y\right) x=\frac{a b}{4} .
$$

Then the solutions $T_{n m}^{\text {first }}$ for the first impact of duration $\tau$ are found similar to Eq. (46),

$$
T_{n, m}^{\text {first }}(t)=\frac{P X_{n m}\left(x_{0}, y_{0}\right)}{\rho h\left\|X_{n, m}(x)\right\|^{2}} \Phi_{n, m}(t, \tau, 0),
$$

leading to the following Fourier series (all Eqs. (10), (75), (76) are employed):

$$
w^{\mathrm{first}}(x, y, t)=\sum_{n=1}^{\infty} \sum_{m=1}^{\infty} \frac{P X_{n m}\left(x_{0}, y_{0}\right)}{\rho h\left\|X_{n, m}(x)\right\|^{2}} \Phi_{n, m}(t, \tau, 0) X_{n, m}(x, y)
$$




$$
\begin{aligned}
= & \sum_{n=1}^{\infty} \sum_{m=1}^{\infty} \frac{4 P a b \sin \left(\frac{\pi n}{a} x_{0}\right) \sin \left(\frac{\pi m}{b} y_{0}\right)}{N \pi^{2}\left(n^{2} b^{2}+m^{2} a^{2}\right)} \sin \left(\frac{\pi n}{a} x\right) \sin \left(\frac{\pi m}{b} y\right) \times \\
& \times\left(\left[1-\cos \left(\omega_{n m}(t)\right)\right] H(t)-\left[1-\cos \left(\omega_{n m}(t-\tau)\right)\right] H(t-\tau)\right),
\end{aligned}
$$

with eigenfrequencies

$$
\omega_{n, m}=\pi \sqrt{\frac{N}{\rho h}\left[\left(\frac{n}{a}\right)^{2}+\left(\frac{m}{b}\right)^{2}\right]}, \quad n, m=1,2,3, \ldots
$$

The second counter impact is generated at the time $\tau_{11}$ according to the definition of the corresponding eigenperiods $P_{n m}$ in Eq. (9):

$$
P_{n m}=\frac{2 \pi}{\omega_{n m}}=2 a b \cdot \sqrt{\frac{\rho}{N\left(n^{2} b^{2}+m^{2} a^{2}\right)}} .
$$

The corresponding functions $T_{n m}^{\text {second }}$ (opposite direction) are defined similar to Eq. (47):

$$
T_{n, m}^{\text {second }}(t)=\frac{-P X_{n m}\left(x_{0}, y_{0}\right)}{\rho h\left\|X_{n, m}(x)\right\|^{2}} \Phi_{n, m}\left(t, \tau, \tau_{n m}\right)
$$

in which the response functions are calculated at the time $\tau_{n m}$ consequently, and the whole series is activated at time $\tau_{11}$. For the double impact in the opposite direction, it is required that the condition in Eq. (19) from the Property 5 for the response function is fulfilled, see also the rule described in Sect. 3.2 for the arbitrary structure. The counter impact satisfying the wave cancellation condition should be applied at the integer number of eigen periods. In this case, this proportionality is fulfilled if we chose $\tau_{n m}=k P_{n m}$ for the selected integer number $k=1,2,3 \ldots$ and apply the second impact at the time $\tau_{n m}=k P_{11}$.

The following Fourier series for the second impact is written as:

$$
\begin{aligned}
w^{\text {second }}(x, y, t)= & \sum_{n=1}^{\infty} \sum_{m=1}^{\infty} \frac{-P X_{n m}\left(x_{0}, y_{0}\right)}{\rho h\left\|X_{n, m}(x)\right\|^{2}} \Phi_{n, m}\left(t, \tau, \tau_{n m}\right) X_{n, m}(x, y) H\left(t-\tau_{11}\right) \\
= & \sum_{n=1}^{\infty} \sum_{m=1}^{\infty} \frac{-4 P a b \sin \left(\frac{\pi n}{a} x_{0}\right) \sin \left(\frac{\pi m}{b} y_{0}\right)}{N \pi^{2}\left(n^{2} b^{2}+m^{2} a^{2}\right)} \sin \left(\frac{\pi n}{a} x\right) \sin \left(\frac{\pi m}{b} y\right) H\left(t-\tau_{11}\right) . \\
& \times\left(\left[1-\cos \left(\omega_{n m}\left(t-\tau_{n m}\right)\right] H\left(t-\tau_{n m}\right)-\left[1-\cos \left(\omega_{n m}\left(t-\tau_{n m}-\tau\right)\right)\right] H\left(t-\tau_{n m}-\tau\right)\right) .\right.
\end{aligned}
$$

\subsection{Numerical example: counter impact in the opposite direction}

As a numerical example, we will show an impact in the opposite direction only without the loss of generality. The thin membrane of size $a=1 \mathrm{~m}, b=2 \mathrm{~m}$ and thickness $h=0.01 \mathrm{~m}$ is subjected to pretension stress with $N=1000 \mathrm{~N} \mathrm{~m}^{-1}$, density is $\rho=7800 \mathrm{~kg} \mathrm{~m}^{-3}$. According to these values the first eigen period is calculated via Eq. (80) as $P_{11}=4.996 \times 10^{-1} \mathrm{~s}$. The membrane is impacted at point $x_{0}=0.25 \mathrm{~m}, y_{0}=0.35 \mathrm{~m}$ with the force $F=10 \mathrm{~N}$ of the duration $\tau=0.2 P_{11}=9.991 \times 10^{-2} \mathrm{~s}$. Results for the single impact is shown in Fig. 12 (red line). The second impact is applied at the time equals to four periods $\tau_{11}=4 \times T_{11}=1.998 \mathrm{~s}$ and further $\tau_{n m}=4 T_{n m}$. Twenty terms for both directions $n=20, m=20$ are taken in the double Fourier series for $w(x, y, t)=w^{\text {first }}(x, y, t)+w^{\text {second }}(x, y, t)$ in Eqs. (78)-(82). The result for the double impact is shown in Fig. 12 as green line-green and red lines are overlapping at $0 \leq t \leq \tau_{11}$. In addition in this Figure, computational results only for the second impact, see Eq. (82) is shown as blue crossed line.

It can be seen that the first wave generated by the first impact is fully canceled by the second counter impact and the membrane is standing still after the time $t=\tau+\tau_{11}$ (results are computed at the impact point $\left(x_{0}, y_{0}\right)$.

Remark 4 (Double impact on the Kirchhoff plate) Another very similar example for the double impact problem possessing analytical solutions can be constructed for the simply supported plate with respect to the Kirchhoff plate theory theory, see Appendix A.4. 


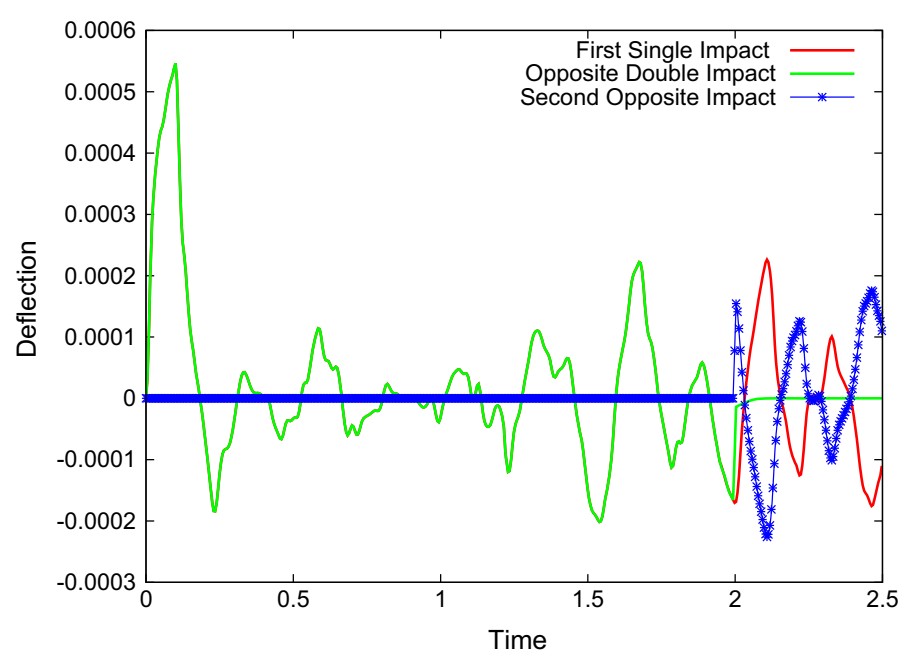

Fig. 12 Vertical displacement of the membrane $w$ at the point $x 0, y 0$ of applied force $P$. The double opposite impact. The membrane is standing still after the counter impact at time $t=\tau_{11}+\tau=2.098 \mathrm{~s}$

\section{Conclusion}

The current contribution studied the wave cancellation conditions for the double impact system in an arbitrary structure. The first impact of a final duration $\tau$ is applied at a certain point and is causing the first wave, the second counter impact is applied in a certain time $\tau_{1}$ such that the vibrations are fully cancelled. First, this phenomenon is studied in detail in the closed form for the double impact in 1D bar. It is possible to find the time of application for the counter impact depending on the $k$ th eigenvalue of the structure as well as the time of its duration such that the vibration is disappearing. A response function is introduced in order to calculate all parameters of the double impact leading to the wave cancellation conditions. The result is generalized for any arbitrary homogeneous linear non-dissipative mechanical structure described by a semi-elliptic operator $L u$. It is shown by using the properties of the response function, that for a general structure the counter impact can be generated in both the same and the opposite direction to the first impact. Wave cancellation conditions are formulated as specification of the second impact of duration $\tau$, applied after an integer number $k$ of the first eigenperiod $P_{1}$ for the same impact direction, or applied after uneven number divided by two $\frac{2 k+1}{2}$ of the fist eigenperiod $P_{1}$. The vibrations are fully disappearing within the application time $\tau$ of the second impact (i.e. fully canceled after the time $t=\tau+\tau_{1}$ ). Numerically it has been illustrated for the set of problems possessing analytical solutions in Fourier series: simply supported and clamped beams, a membrane. The solution for the simply supported Kirchhoff is also presented. The amplitude for the counter impact for any arbitrary structure depends on higher order periods $P_{n}$, though the contributions of the higher order terms is rather negligible.

Summarizing the development we can see the three major fields of potential applications of the general result:

1. As a set of perfect verification examples in computational contact mechanics [19] in order to test numerical time integration schemes especially with energy conservation properties [7,28]. It is well known, that these schemes are verified on the example with a-priory conserved energy and are required the long time integration in order to test the energy conservation properties, see [3]. Providing a set of examples with both wave and energy cancellation properties will allow to decrease the time integration for verification.

2. The result formulated in Sect. 3 as wave cancellation conditions for an arbitrary structure in differential forms can be straightforwardly transferred into any convenient numerical methods in solid mechanics: finite element method, isogeometric method etc.

3. The structure of the opposite impact causing the cancellation is fully recovered by the first one, therefore these result can be directly employed during engineering design process for various impacting devices (hammering etc.) to prevent the recoil. 
Open Access This article is licensed under a Creative Commons Attribution 4.0 International License, which permits use, sharing, adaptation, distribution and reproduction in any medium or format, as long as you give appropriate credit to the original author(s) and the source, provide a link to the Creative Commons licence, and indicate if changes were made. The images or other third party material in this article are included in the article's Creative Commons licence, unless indicated otherwise in a credit line to the material. If material is not included in the article's Creative Commons licence and your intended use is not permitted by statutory regulation or exceeds the permitted use, you will need to obtain permission directly from the copyright holder. To view a copy of this licence, visit http://creativecommons.org/licenses/by/4.0/.

\section{A Appendix}

A.1 Fourier series method for the solution of the homogeneous PDE

The homogeneous equation for the PDE in Eq. (1) is written as (right side is zero $q(x, t)=0$ )

$$
\frac{\partial^{2} u}{\partial t^{2}}=c^{2} \frac{\partial^{2} u}{\partial x^{2}}
$$

where the parameter $c=\sqrt{\frac{E}{\rho}}$ is the velocity of the wave distribution.

The split of time variable $t$ from the structural variable $x$ is the basis of the standard Fourier series leading to the eigenvalue problem. This split taken as $u(x, t)=X(x) T(t)$ leads to the split of Eq. (83) into a system of two ordinary differential equations

$$
\frac{\ddot{T}}{T} \frac{1}{c^{2}}=\frac{X^{\prime \prime}(x)}{X}=-\lambda^{2}
$$

The equation with the structural variable $x$ from the system (84) together with the boundary conditions gives us the statement of the eigenvalue problem:

- find the set of eigenfunctions $X_{n}(x)$ and eigenvalues $\lambda_{n}$ satisfying the following equation:

$$
X^{\prime \prime}(x)+\lambda^{2} X=0 ;
$$

- and the corresponding boundary conditions (arising from Eq. (2))

$$
X(0)=0 \text { : fixed end, } \frac{\mathrm{dX}}{\mathrm{dx}}(L)=0: \text { free end. }
$$

The well known solution of the eigenvalue problem (85)-(86) is an infinite countable set of the following eigenfunctions $X_{n}(x)$ and eigenvalues $\lambda_{n}$ :

$$
\begin{aligned}
X_{n}(x) & =\sin \left(\lambda_{n} x\right)=\sin \left(\frac{[(2 n+1) \pi] x}{2 L}\right), \\
\lambda_{n} & =\frac{[(2 n+1) \pi] x}{2 L} .
\end{aligned}
$$

This set of eigenfunctions is forming an orthogonal basis in the Hilbert space $\{\mathbf{H}: y=f(x), 0 \leq x \leq L\}$ equipped with the following scalar product:

$$
\langle f(x) \cdot g(x)\rangle=\int_{0}^{L} f(x) g(x) \mathrm{d} x .
$$

Thus, the orthogonality of the eigenfunctions $X_{n}(x)$ is the fulfillment of the following conditions:

$$
\left\langle X_{n}(x) \cdot X_{k}(x)\right\rangle=\int_{0}^{L} X_{n}(x) \cdot X_{k}(x) \mathrm{d} x=\delta_{k n}\left\|X_{k}(x)\right\|^{2},
$$

where $\delta_{k n}$ is the Kronecker delta and $\left\|X_{k}(x)\right\|$ is the norm of the eigenfunction.

The full solution of the homogeneous PDE (83) is given, therefore, in the form of a Fourier series as

$$
u(x, t)=\sum_{n=0}^{\infty} T_{n}(t) X_{n}(x)=\sum_{n=0}^{\infty} T_{n}(t) \sin \left(\frac{(2 n+1) \pi}{2 L} x\right),
$$

where all time dependent functions $T_{n}(t)$ should satisfy the corresponding initial conditions. 
A.2 Lemma 1: solution of non-homogeneous ODE via the convolution with a fundamental function

Lemma 1 The solution of the non-homogeneous $O D E$

$$
\ddot{T}(t)+\omega^{2} T(t)=f(t)
$$

is derived in the form of a convolution with the fundamental solution $T_{f}(t)$ as

$$
T(t)=T_{f}(t) * f(t)=\int_{-\infty}^{\infty} T_{f}(\tau) f(t-\tau) \mathrm{d} \tau .
$$

The fundamental solution $T_{f}(t)$ is a solution in the form of generalized functions:

$$
\ddot{T}_{f}(t)+\omega^{2} T_{f}(t)=\delta(t) .
$$

This solution is found as the solution of the following initial value problem for the ODE:

$$
\begin{aligned}
& \ddot{T}_{h}(t)+\omega^{2} T_{h}(t)=0 ; \\
& T_{h}(0)=0 ; \quad \frac{\mathrm{d} T_{h}}{\mathrm{~d} t}=1,
\end{aligned}
$$

and is written as

$$
T_{f}(t)=T_{h}(t) H(t)=\frac{\sin (\omega t)}{\omega} H(t)
$$

where $\frac{\sin (\omega t)}{\omega}$ is the solution of the initial value problem in Eqs. (94)-(95) and $H(t)$ is the Heaviside function.

A.3 Fourier series method for the solution of the non-homogeneous PDE

The solution of the non-homogeneous PDE (1) can be found in the form Eq. (90), satisfying the boundary conditions (2) (because of the choice of eigenfunctions). The standard transformation of the PDE, namely a scalar product (Eq. 88) with eigenfunctions $X_{n}(x)$ in the corresponding Hilbert space leads to due the orthogonality property in Eq. (89) to the set of ordinary differential equations (ODE) for time dependent functions $T_{n}(t)$ :

$$
\ddot{T}_{n}(t)+c^{2} \lambda_{n}^{2} T_{n}(t)=\frac{\left\langle q(x, t) \cdot X_{n}(x)\right\rangle}{\rho A\left\|X_{k}(x)\right\|^{2}} .
$$

For the problem in Eq. (90), the norm of the eigenfunctions is computed as

$$
\left\|X_{k}(x)\right\|^{2}=\int_{0}^{L}\left(\sin \left(\frac{(2 k+1) \pi}{2 L} x\right)\right)^{2} \mathrm{~d} x=\frac{L}{2} .
$$

Each of Eqs. (97) should satisfy the initial conditions arising from the integration of initial conditions in Eq. (3) for the original PDE (scalar product in the corresponding Hilbert space):

$$
T_{n}(0)=0 ; \frac{\mathrm{d} T_{n}}{\mathrm{~d} t}(0)=0 .
$$

The solution of the non-homogeneous ODE (97) can be obtained in the form of a convolution with the fundamental solution employing Lemma 1 in A.2, see more about methods in [13,33,34]:

$$
T_{n}(t)=\frac{\sin \left(\omega_{n} t\right)}{\omega_{n}} H(t) * \frac{\left\langle q(x, t) \cdot X_{n}(x)\right\rangle}{\rho A\left\|X_{k}(x)\right\|^{2}} H(t)
$$

with $\omega_{n}=c \lambda_{n}$. 
A.4 Solution of the double impact problem for the simply supported Kirchhoff plate

Consider a rectangular plate with size $a \times b$ and thickness $h$, made of elastic material with elasticity modulus $E$, Poisson's ratio $v$ and constant density $\rho$. The kinematic of deformation is satisfying the Kirchhoff theory. The plate is simply supported. At time $t=0$ the constant impacting load $P$ of duration $\tau$ is applied at the point $x_{0}, y_{0}$. The dynamic motion is described by the vertical displacement function $w(x, y, t)$, which, according to the Kirchhoff-Love plate hypothesis, is satisfying the following equation:

$$
D \triangle \Delta w+\rho h \frac{\partial^{2} w}{\partial t^{2}}=q(x, y, t) .
$$

The plate stiffness is defined as

$$
D=\frac{E h^{3}}{12\left(1-v^{2}\right)} .
$$

The distributed force $q(x, t)$ is represented via the Dirac function as $q=P(t) \delta\left(x-x_{0}\right) \delta\left(y-y_{0}\right)$. The double Laplace differential operator is defined as

$$
\triangle \triangle w(x, y, t) \equiv \frac{\partial^{4} w}{\partial x^{4}}+2 \frac{\partial^{4} w}{\partial x^{2} \partial y^{2}}+\frac{\partial^{4} w}{\partial y^{4}} .
$$

The function $w(x, y, t)$ for the simply supported plate should satisfy the following boundary conditions:

- The displacements are zero at all boundary lines:

$$
w(0, y, t)=0, w(a, y, t)=0, w(x, 0, t)=0, w(x, b, t)=0 .
$$

- The corresponding bending moments are zero at all boundary lines:

$$
M_{y}(0, y, t)=0, M_{y}(a, y, t)=0, M_{x}(x, 0, t)=0, M_{x}(x, b, t)=0 ;
$$

which are transformed according to the Kirchhoff plate theory to the following equations:

$$
\frac{\partial^{2} w(0, y, t)}{\partial x^{2}}=0, \frac{\partial^{2} w(a, y, t)}{\partial x^{2}}=0, \frac{\partial^{2} w(x, 0, t)}{\partial y^{2}}=0, \frac{\partial^{2} w(x, b, t)}{\partial y^{2}}=0 .
$$

It is well known that in this simplest case the eigenfunctions of the corresponding eigenvalue problem

$$
\frac{X^{I V}(x)}{X(x)}+2 \frac{X^{\prime \prime}(x) Y^{\prime \prime}(y)}{X(x) Y(y)}+\frac{Y^{I V}(y)}{Y(y)}=\lambda^{4}
$$

are fully coinciding to those for the membrane in Eq. (75) with eigenvalues

$$
\lambda_{n, m}^{4}=\left(\frac{\pi n}{a}\right)^{4}+2\left(\frac{\pi n}{a}\right)^{2}\left(\frac{\pi m}{b}\right)^{2}+\left(\frac{\pi m}{b}\right)^{4}, \quad n, m=1,2,3, \ldots
$$

The first impact is represented by the following Fourier series

$$
\begin{aligned}
w^{\mathrm{first}}(x, y, t)= & \sum_{n=1}^{\infty} \sum_{m=1}^{\infty} \frac{P X_{n m}\left(x_{0}, y_{0}\right)}{\rho h\left\|X_{n, m}(x)\right\|^{2}} \Phi_{n, m}(t, \tau, 0) X_{n, m}(x, y) \\
= & \sum_{n=1}^{\infty} \sum_{m=1}^{\infty} \frac{4 P a^{3} b^{3} \sin \left(\frac{\pi n}{a} x_{0}\right) \sin \left(\frac{\pi m}{b} y_{0}\right)}{D \pi^{4}\left(n^{2} b^{2}+m^{2} a^{2}\right)^{2}} \sin \left(\frac{\pi n}{a} x\right) \sin \left(\frac{\pi m}{b} y\right) . \\
& \times\left(\left[1-\cos \left(\omega_{n m}(t)\right)\right] H(t)-\left[1-\cos \left(\omega_{n m}(t-\tau)\right)\right] H(t-\tau)\right),
\end{aligned}
$$

with eigenfrequencies

$$
\omega_{n, m}^{2}=\pi^{4} \frac{D}{\rho h}\left[\left(\frac{n}{a}\right)^{2}+\left(\frac{m}{b}\right)^{2}\right]^{2}, \quad n, m=1,2,3, \ldots
$$


The second impact is represented by the following Fourier series:

$$
\begin{aligned}
w^{\text {second }}(x, y, t)= & \sum_{n=1}^{\infty} \sum_{m=1}^{\infty} \frac{ \pm P X_{n m}\left(x_{0}, y_{0}\right)}{\rho h\left\|X_{n, m}(x)\right\|^{2}} \Phi_{n, m}\left(t, \tau, \tau_{n m}\right) X_{n, m}(x, y) H\left(t-\tau_{11}\right) \\
= & \sum_{n=1}^{\infty} \sum_{m=1}^{\infty} \frac{ \pm 4 P_{1} a^{3} b^{3} \sin \left(\frac{\pi n}{a} x_{0}\right) \sin \left(\frac{\pi m}{b} y_{0}\right)}{D \pi^{4}\left(n^{2} b^{2}+m^{2} a^{2}\right)^{2}} \sin \left(\frac{\pi n}{a} x\right) \sin \left(\frac{\pi m}{b} y\right) H\left(t-\tau_{11}\right) \\
& \times\left(\left[1-\cos \left(\omega_{n m}\left(t-\tau_{n m}\right)\right] H\left(t-\tau_{n m}\right)\right.\right. \\
& \left.-\left[1-\cos \left(\omega_{n m}\left(t-\tau_{n m}-\tau\right)\right)\right] H\left(t-\tau_{n m}-\tau\right)\right)
\end{aligned}
$$

The cancellation conditions are as follows:

- For the double impact in the opposite direction, i.e. $P_{1}=-P$ in Eq. (111):

The second impact starts at $\tau_{1}=k P_{11}$ and all $\tau_{n m}=k P_{n m}=k \frac{2 \pi}{\omega_{n m}}$ for any integer $k=1,2,3 \ldots$

- For the double impact in the same direction, i.e. $P_{1}=P$ in Eq. (111):

The second impact starts at $\tau_{1}=\frac{2 k+1}{2} P_{11}$ and all $\tau_{n m}=\frac{2 k+1}{2} P_{n m}=\frac{2 k+1}{2} \frac{2 \pi}{\omega_{n m}}$ for any integer $k=1,2,3 \ldots$.

\section{References}

1. Auersch, L.: Response to harmonic wave excitation of finite or infinite elastic plates on a homogeneous or layered half-space. Comput. Geotech. 51, 50-59 (2013). https://doi.org/10.1016/j.compgeo.2013.02.001

2. Babitski, V., Krupenin, V.: Vibration of Strongly Nonlinear Discontinuous Systems. Springer, Berlin (2001)

3. Betsch, P., Steinmann, P.: Conservation properties of a time FE method. Part I: Time-stepping schemes for n-body problems. Int. J. Numer. Methods Eng. 49(5), 599-638 (2000). https://doi.org/10.1002/1097-0207(20001020)49:5<599::AIDNME960>3.0.CO;2-9

4. Brogliato, B.: Impacts in Mechanical Systems: Analysis and Modeling. Springer, Berlin (2000)

5. Chen, Y., McFarland, D.M., Spencer, B.F., Bergman, L.A.: Exact solution of free vibration of a uniform tensioned beam combined with both lateral and rotational linear sub-systems. J. Sound Vib. 341, 206-221 (2015). https://doi.org/10.1016/j. jsv.2014.12.013

6. Chung, J., Crocker, M.: The effect of wave cancellation on the radiation resistance of a simply-supported baffled beam. J. Sound Vib. 40(3), 381-391 (1975). https://doi.org/10.1016/S0022-460X(75)81308-3

7. Crisfield, M., Shi, J.: An energy conserving co-rotational procedure for non-linear dynamics with nite elements. Nonlinear Dyn. 9, 37-52 (1996)

8. Cui, D., Hu, H.: Primary resonance of lateral vibration of a heated beam with an axial stick-slip-stop boundary. J. Sound Vib. 339, 230-246 (2015). https://doi.org/10.1016/j.jsv.2014.10.042

9. Dingyue, C., Xia, L., Hongsheng, C., Hui, X., Jianjia, D.: Study of vibration suppression in discrete domain. Acta Mech. 158(1), 57-66 (2002). https://doi.org/10.1007/BF01463169

10. Filippov, A.: Differential Equations with Discontinuous Right-Hand Sides. Kluwer Academic Publishers, Berlin (1988)

11. Futhazar, G., Parnell, W.J., Norris, A.N.: Active cloaking of flexural waves in thin plates. J. Sound Vib. 356, 1-19 (2015). https://doi.org/10.1016/j.jsv.2015.06.023

12. Goldsmith, W.: Impact: The Theory and Physical Behavior of Colliding. Dover Publications, Mineola (2001)

13. Hansen, C., Snyder, S., Qiu, X., Brooks, L., Moreau, D.: Control of Noise and Vibration, 2nd edn. CRC Press, London (2012)

14. Huang, Q., Xu, Y.: Active cancellation stealth analysis based on interrupted-sampling and convolution modulation. Opt. Int. J. Light Electron Opt. 127(7), 3499-3503 (2016). https://doi.org/10.1016/j.ijleo.2015.12.129

15. Ibrahim, R.: Vibro-Impact Dynamics: Modeling, Mapping and Applications. Springer, Berlin (2009)

16. Ibrahim, R.A.: Recent advances in vibro-impact dynamics and collision of ocean vessels. J. Sound Vib. 333(23), 5900-5916 (2014). https://doi.org/10.1016/j.jsv.2014.02.006

17. Jones, N.: Structural Impact. Cambridge University Press, Cambridge (1997)

18. Kalnins, A.: Vibration: Beams, Plates, and Shells. Dowden, Hutchinson and Ross, Stroudsburg (1976)

19. Konyukhov, A., Schweizerhof, K.: Computational Contact Mechanics: Geometrically Exact Theory for Arbitrary Shaped Bodies. Springer, Heidelberg (2013)

20. Kuo, S.M., Morgan, D.R.: Active noise control: a tutorial review. Proc. IEEE 87(6), 943-973 (1999)

21. Lee, W., Lee, H., Kim, Y.Y.: Experiments of wave cancellation with elastic phononic crystal. Ultrasonics 72, 128-133 (2016). https://doi.org/10.1016/j.ultras.2016.08.001

22. Lu, J.F., Sha, X., Wu, J.B.: Resonance and cancellation phenomena caused by equidistant moving loadings in a periodic structure: a pile-supported periodic viaduct. Eur. J. Mech. A Solids 59, 114-123 (2016). https://doi.org/10.1016/j.euromechsol. 2016.03.010 
23. Malookani, R.A., van Horssen, W.T.: On resonances and the applicability of Galerkin's truncation method for an axially moving string with time-varying velocity. J. Sound Vib. 344, 1-17 (2015). https://doi.org/10.1016/j.jsv.2015.01.051

24. Museros, P., Moliner, E., Martínez-Rodrigo, M.: Free vibrations of simply-supported beam bridges under moving loads: maximum resonance, cancellation and resonant vertical acceleration. J. Sound Vib. 332(2), 326-345 (2013). https://doi.org/ 10.1016/j.jsv.2012.08.008

25. Samagassi, S., Khamlichi, A., Driouach, A., Jacquelin, E.: Reconstruction of multiple impact forces by wavelet relevance vector machine approach. J. Sound Vib. 359, 56-67 (2015). https://doi.org/10.1016/j.jsv.2015.08.014

26. Schoeftner, J., Krommer, M.: Single point vibration control for a passive piezoelectric Bernoulli-Euler beam subjected to spatially varying harmonic loads. Acta Mech. 223(9), 1983-1998 (2012). https://doi.org/10.1007/s00707-012-0686-0

27. Sharma, B., Sun, C.: Local resonance and Bragg bandgaps in sandwich beams containing periodically inserted resonators. J. Sound Vib. 364, 133-146 (2016). https://doi.org/10.1016/j.jsv.2015.11.019

28. Simo, J., Tarnow, W.: The discrete energy-momentum method. Conserving algorithms for nonlinear elastodynamics. J. Appl. Math. Phys. 43(5), 757-792 (1992)

29. Sinha, A.: Vibration of Mechanical Systems. Cambridge University Press, Cambridge (2010)

30. Teo, Y.R., Fleming, A.J.: Optimal integral force feedback for active vibration control. J. Sound Vib. 356, 20-33 (2015). https://doi.org/10.1016/j.jsv.2015.06.046

31. Tuer, K., Duquette, A., Golnaraghi, M.: Vibration control of a flexible beam using a rotational internal resonance controller, part I: theoretical development and analysis. J. Sound Vib. 167(1), 41-62 (1993). https://doi.org/10.1006/jsvi.1993.1320

32. Venkata Rao, K., Raja, S., Munikenche Gowda, T.: Finite element modelling and vibration control study of active plate with debonded piezoelectric actuators. Acta Mech. 225(10), 2923-2942 (2014). https://doi.org/10.1007/s00707-014-1207-0

33. Vladimirov, V.S.: A Collection of Problems on the Equations of Mathematical Physics. Springer, Berlin (1986)

34. Vladimirov, V.S.: Methods of the Theory of Generalized Functions. CRC Press, Boca Raton (2002)

35. Yang, Y.B., Lin, C.L., Yau, J.D., Chang, D.W.: Mechanism of resonance and cancellation for train-induced vibrations on bridges with elastic bearings. J. Sound Vib. 269(1-2), 345-360 (2004). https://doi.org/10.1016/S0022-460X(03)00123-8

36. Yang, Y.B., Yau, J.D., Hsu, L.C.: Vibration of simple beams due to trains moving at high speeds. Eng. Struct. 19(11), 936-944 (1997). https://doi.org/10.1016/S0141-0296(97)00001-1

37. Yau, J.D., Yang, Y.B.: Vibration reduction for cable-stayed bridges traveled by high-speed trains. Finite Elem. Anal. Des. 40(3), 341-359 (2004). https://doi.org/10.1016/S0168-874X(03)00051-9

38. Zhang, W., Chen, L.Q.: Vibration control of an axially moving string system: wave cancellation method. Appl. Math. Comput. 175(1), 851-863 (2006). https://doi.org/10.1016/j.amc.2005.08.007

39. Zhang, Y.H., Xie, S.L., Zhang, X.N.: Vibration control of a simply supported cylindrical shell using a laminated piezoelectric actuator. Acta Mech. 196(1), 87-101 (2008). https://doi.org/10.1007/s00707-007-0501-5

40. Zuo, S., Liu, Y., Zhang, K., Hu, G.: Wave boundary control method for vibration suppression of large net structures. Acta Mech. 230(10), 3439-3456 (2019). https://doi.org/10.1007/s00707-019-02464-1

Publisher's Note Springer Nature remains neutral with regard to jurisdictional claims in published maps and institutional affiliations. 